\title{
Taxonomy of Aspidosperma Mart. (Apocynaceae, Rauvolfioideae) in the State of Pará, Northern Brazil
}

\author{
Andreza Stephanie de Souza Pereira ${ }^{1,3}$, André Olmos Simões ${ }^{2}$ \& João Ubiratan Moreira dos Santos ${ }^{1}$ \\ ${ }^{1}$ Programa de Pós-graduação em Ciências Biológicas - Botânica Tropical, Universidade Federal Rural da \\ AmazônialMuseu Paraense Emílio Goeldi, Belém, PA, Brazil. \\ ${ }^{2}$ Departamento de Biologia Vegetal, Instituto de Biologia, Universidade Estadual de Campinas, Campinas, \\ SP, Brazil. \\ ${ }^{3}$ Corresponding author: Andreza Stephanie de Souza Pereira, e-mail: andrezapereira_bio@yahoo.com.br
}

PEREIRA, A.S.S., SIMÕES, A.O., SANTOS, J.U.M. Taxonomy of Aspidosperma Mart. (Apocynaceae, Rauvolfioideae) in the State of Pará, Northern Brazil. Biota Neotropica. 16(2): e20150080. http://dx.doi. org/10.1590/1676-0611-BN-2015-0080

\begin{abstract}
Aspidosperma Mart. is one of the most important genera from Apocynaceae Juss. occurring in Brazil. It is present in many plant formations and has species with both medicinal and economic value. From a taxonomic point of view, many of its species are difficult to identify, especially those occurring in the Amazonian region. In order to complement the information already available with regard to the taxonomy and distribution of the genus, the goal of this work was to provide a taxonomic study of the Aspidosperma species occurring in the state of Pará, northern Brazil. This study was based on the analysis of vouchers from the BHCB, HBRA, IAC, IAN, INPA, MG, R, RB and UEC herbaria, as well as scanned images of vouchers from Brazilian and foreign herbaria, and specimens collected in the field. Species identification was confirmed by analysis of protologues and types (or images). We present taxonomic descriptions, illustrations and one identification key for the 20 species of Aspidosperma found in Pará, with the Baixo Amazonas Mesoregion presenting the largest number of species (17), and the Metropolitana de Belém Mesoregion presenting the smallest number of species (3). Aspidosperma eteanum, A. oblongum, A. salgadense (endemic of Pará) and A. sandwithianum are accepted as species here. Aspidosperma cuspa is a new record for Pará. The fruits of Aspidosperma eteanum and $A$. salgadense, and the seeds of $A$. eteanum, have been described and illustrated for the first time.

Keywords: Aspidospermeae, diversity, Eastern Amazon, floristic, morphology.
\end{abstract}

PEREIRA, A.S.S., SIMÕES, A.O., SANTOS, J.U.M. Taxonomia de Aspidosperma Mart. (Apocynaceae, Rauvolfioideae) no Estado do Pará, Brasil. Biota Neotropica. 16(2): e20150080. http://dx.doi.org/10.1590/ 1676-0611-BN-2015-0080

Resumo: Dentre os gêneros de Apocynaceae Juss. ocorrentes no Brasil, Aspidosperma Mart. é um dos mais importantes. Está presente em várias formações vegetais e apresenta espécies com valor medicinal e econômico. Do ponto de vista taxonômico, várias de suas espécies são de difícil identificação, principalmente as que ocorrem na Amazônia. Com o intuito de complementar as informações disponíveis sobre a taxonomia e a distribuição do gênero, este trabalho teve por objetivo realizar estudos taxonômicos com as espécies de Aspidosperma ocorrentes no estado do Pará, Brasil. Este estudo foi baseado na análise de exsicatas dos herbários BHCB, HBRA, IAC, IAN, INPA, MG, R, RB e UEC, assim como de imagens digitalizadas de exsicatas de herbários nacionais e estrangeiros, e de materiais coletados. A identificação das espécies foi confirmada por meio de consulta aos protólogos e tipos (ou imagens). São apresentadas descrições, ilustrações e uma chave de identificação para as 20 espécies de Aspidosperma encontradas no Pará, com a Mesorregião do Baixo Amazonas apresentando um maior número de espécies (17), e a Mesorregião Metropolitana de Belém apresentando um menor número de espécies (3). As espécies Aspidosperma eteanum, A. oblongum, A. salgadense (endêmica do Pará) e $A$. sandwithianum são aqui aceitas. Aspidosperma cuspa é uma nova ocorrência para o Pará. Os frutos de Aspidosperma eteanum e $A$. salgadense, e as sementes de $A$. eteanum, estão sendo descritos e ilustrados pela primeira vez.

Palavras-chave: Aspidospermeae, diversidade, Amazônia Oriental, florística, morfologia. 


\section{Introduction}

Apocynaceae Juss. is one of the five families of the order Gentianales, and is one of the ten largest Angiosperm families in the world (APG III 2009, Rapini 2012, Morokawa et al. 2013). According to the most recent classifications, the family is divided into five subfamilies: Rauvolfioideae, Apocynoideae, Periplocoideae, Secamonoideae and Asclepiadoideae (Endress \& Bruyns 2000, Endress et al. 2007, 2014). Apocynaceae has a cosmopolitan distribution, comprising of 366 genera and ca. 5100 species (Matozinhos \& Konno 2011, Nazar et al. 2013, Endress et al. 2014). In Brazil, Apocynaceae is the tenth largest Angiosperm family, with members belonging to subfamilies Rauvolfioideae, Apocynoideae and Asclepiadoideae (Kinoshita \& Simões 2005, Rapini et al. 2010).

Among the Brazilian genera of Apocynaceae, Aspidosperma Mart. (Rauvolfioideae) is one of the most important, being present in many plant formations and is comprised of species with medicinal and economic value (Duarte 1970, Marcondes-Ferreira 1988). Aspidosperma has a Neotropical distribution, occurring from Mexico to Argentina (except for Chile) (MarcondesFerreira \& Kinoshita 1996, Marcondes-Ferreira 1999). According to Marcondes-Ferreira (1999), about 44 species are currently recognized in the genus. Thirty-nine of these are reported to occur in Brazil (10 endemic), with the greatest concentration found in the Amazon (25 species) (Marcondes-Ferreira 1999, Koch et al. 2015).

Aspidosperma was created by Martius (1824a) with five species. The genus is characterized by an arboreal or shrubby habit, latex whitish to reddish, leaves alternate, rarely opposite or whorled, flowers with the style-head fusiform to globose, anthers free from the style-head, follicles woody and seeds winged (Woodson 1951, Marcondes-Ferreira 1988, Simões \& Kinoshita 2002, Kinoshita \& Simões 2005, Morokawa et al. 2013). In the Brazilian Amazon region, most species of Aspidosperma are popularly known as "carapanaúba" or "araracanga", whereas in other regions of the country they are mainly known as "peroba", "guatambu" or "pereiro" (Koch et al. 2015).

Some species of the genus have medicinal value, such as Aspidosperma ramiflorum Müll.Arg., which has antileishmanial properties, and $A$. excelsum Benth., which has antimalarial properties (Pereira et al. 2007, Oliveira et al. 2009). Other species have economic importance in industry and timber production, and are used as ornamental plants (Lorenzi 2008, 2009, Silva 2013). For example, the wood of the "perobas", Aspidosperma cylindrocarpon Müll.Arg. and A. polyneuron Müll.Arg., are widely used in construction and in landscaping (Lorenzi 2008, 2009).

Regarding to group's taxonomy, some of the most relevant work was done by de Candolle (1844), Müller-Argoviensis (1860), Schumann (1895), Pichon (1947), Woodson (1951), MarcondesFerreira (1988) and Marcondes-Ferreira \& Kinoshita (1996). The latter proposed the infrageneric division that is currently accepted for Aspidosperma, dividing the genus in two subgenera A. subgenus Aspidosperma (comprising nine sections) and $A$. subgenus Coutinia (Vell.) Marc.-Ferr. (without further subdivisions) (Marcondes-Ferreira \& Kinoshita 1996). Although Aspidosperma has been a widely studied genus, many of its species are difficult to identify, especially those occurring in the Amazonian region, because some of them are very similar to each other and have overlapping morphological features (Woodson 1951, Marcondes-Ferreira 1988).
These issues justify the importance of taxonomic studies on Aspidosperma to better understand the genus, particularly the Amazonian species. The Brazilian Amazon in the state of Pará is an important area to study Aspidosperma, as it is the second largest state in this region, and according to "Lista de Espécies da Flora do Brasil" (Koch et al. 2015), it is where many of the Amazonian species from this genus occur (17) (Souza et al. 2002, IBGE 2008, Pará 2007, 2010). Furthermore, Aspidosperma is the most represented genus of Apocynaceae family in Pará (Koch et al. 2015). In this context, the goal of this study was to provide a taxonomic study of the Aspidosperma species occurring in the state of Pará, Northern Brazil.

\section{Material and Methods}

\section{Study area}

The study area was located in the state of Pará, Brazil, which has $1,248042 \mathrm{~km}^{2}$, and is the second largest state in the country (Pará 2010). It is located in the North Region of Brazil and in the Amazon Biome (IBGE 2004). The dominant climate is equatorial, with an average temperature of $25^{\circ} \mathrm{C}$ and annual rainfall ranging from 1000 to $4500 \mathrm{~mm}$ (Brasil 1999, IBGE 2004). According to IBGE (1990), the state of Pará is geographically divided into six Mesoregions: Baixo Amazonas, Marajó, Metropolitana de Belém, Nordeste Paraense, Sudeste Paraense and Sudoeste Paraense (Figure 1).

In Pará, there is a predominance of the forest physiognomy, with the Dense Ombrophilous Forest phytoecological region covering most of the region (Baixo Amazonas, Marajó, Metropolitana de Belém, Nordeste Paraense, Sudeste Paraense and Sudoeste Paraense) (IBGE 1990, 2008). There are also areas of Open Ombrophilous Forest (Sudeste Paraense and Sudoeste Paraense), and to a lesser extent, Semideciduous Forest (Sudoeste Paraense), Deciduous Forest (Sudoeste Paraense), regions of Campinarana (Marajó, Sudeste Paraense and Sudoeste Paraense) and Savannah (Baixo Amazonas and Sudeste Paraense) (IBGE 1990, 2008).

\section{Taxonomic treatment}

Herbarium vouchers, scanned images of vouchers from Brazilian and foreign herbaria and specimens collected in the field were analysed in this study. Collections from the BHCB, HBRA, IAC, IAN, INPA, MG, R, RB and UEC herbaria (acronyms according to Thiers (continuously updated)), which hold relevant collections of Amazonian plants, were examined. Online collections from COL, F, INCT - Herbário Virtual da Flora e dos Fungos (speciesLink Network (UFRN, USP)), K, NY and $\mathrm{P}$ herbaria were also examined. The botanical material used was derived from specimens collected in Pará (material examined) and, in general, from specimens collected in the rest of the Amazon, especially in the Brazilian Amazon (additional material).

Plant specimens were collected throughout the year of 2014, from February to September, in Marajó, Metropolitana de Belém and Nordeste Paraense Mesoregions. Specimens were collected and herborized following the usual techniques described by Fidalgo \& Bononi (1984), and vouchers are deposited in the Herbarium of the Museu Paraense Emílio Goeldi (MG).

Species identification was confirmed through the analysis of protologues and types, or through images of types available in 


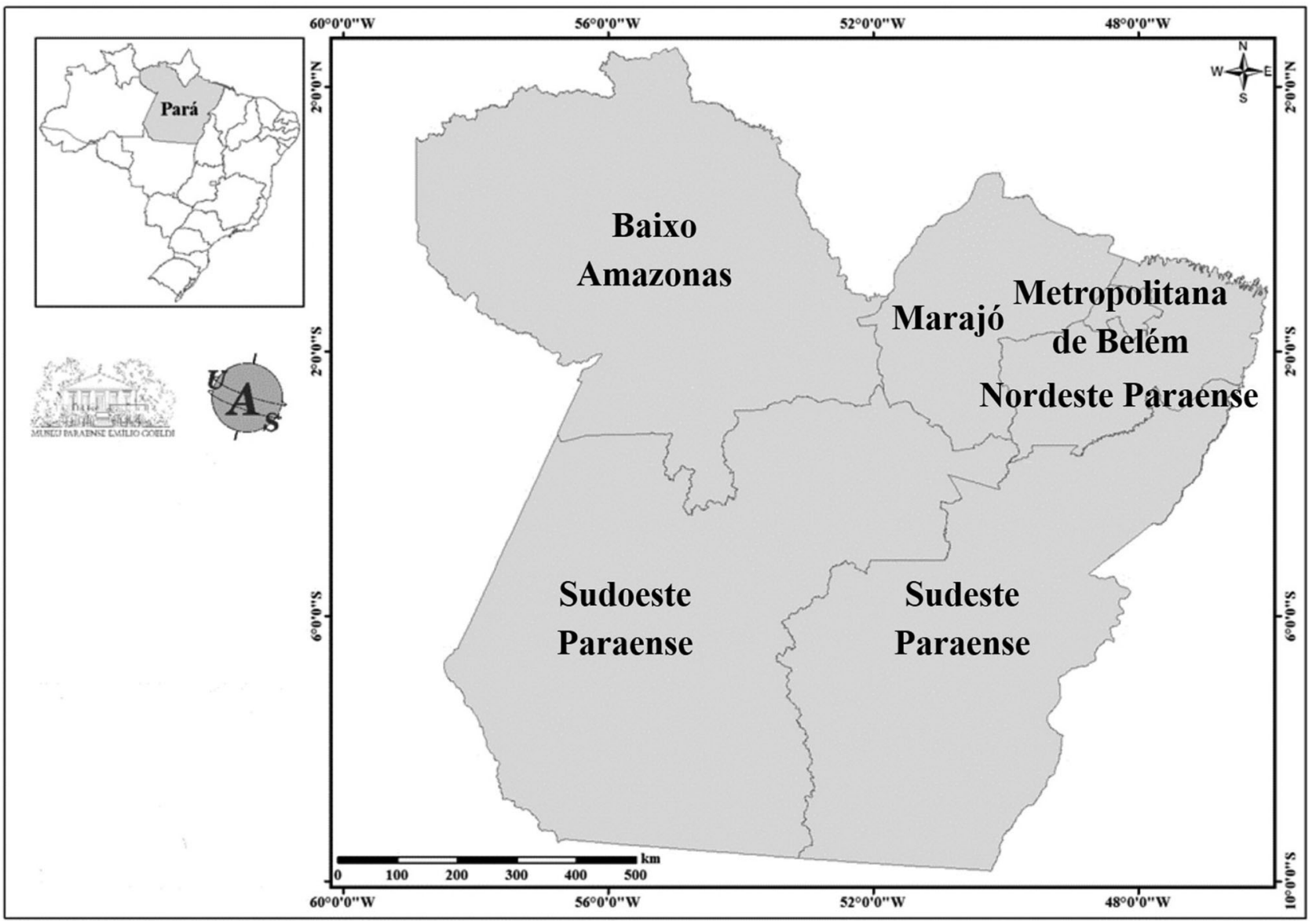

Figure 1. The State of Pará and its Mesoregions, Northern Brazil. Source: MPEG-UAS.

Figura 1. O Estado do Pará e suas Mesorregiões, Brasil. Fonte: MPEG-UAS.

the database "JSTOR Global Plants" (Ithaka 2015), or using the online collections from C, F, G, K, NY, P, U, US and $\mathrm{W}$ herbaria. Spelling of scientific names and information on protologues were checked in the original publications, in the guide "Taxonomic Literature" (Stafleu \& Cowan 1981) and in the databases "Lista de Espécies da Flora do Brasil" (JBRJ 2015), "Tropicos" (Missouri Botanical Garden 2015) and "World Checklist of Selected Plant Families" (WCSP 2015). Author names are abbreviated according to Brummitt \& Powell (1992).

Vegetative structures and follicles were measured with a digital caliper and a ruler, and described with the help of a stereomicroscope. Flowers were rehydrated (when obtained from herbarium specimens), stored in bottles containing $70 \%$ ethanol, dissected, measured with graph paper and described with the help of a stereomicroscope. The terminology used to characterize the vegetative and reproductive structures was based on the works of Woodson (1951), Lawrence (1973), Radford et al. (1974), Rizzini (1977), Marcondes-Ferreira (1988), Ribeiro et al. (1999), Gomes e Cavalcanti (2001), Gomes (2008) and Morokawa et al. (2013). Genus and species descriptions were prepared with the data obtained from analyses on examined material and additional material. Information with regard to geographic distribution, phytoecological regions, phenology and common names was obtained from herbarium specimens, data from field notes and literature.

\section{Results and Discussion}

Aspidosperma Mart., Flora 7(1): 135. 1824. nom. cons. Type: Aspidosperma tomentosum Mart.

Tree, 2-48 m high; trunk straight or tortuous. Branches cylindrical or angular, suberous or not, sparsely to densely lenticellate, velutinous to glabrous, with or without cataphylls covering the buds; latex whitish, orange or reddish. Leaves simple, alternate or, more rarely, subopposite, congested at apex or arranged along the branches; petiole velutinous to glabrous; blade membranaceous to coriaceous, flat or bullate, margin straight or revolute, concolorous or discolorous, venation craspedodromous, brochidodromous, eucamptodromous or reticulodromous, adaxial surface dull or lustrous, greenish, brownish or blackish, velutinous to glabrous, primary vein flat, prominulous or prominent, secondary veins impressed, prominulous or prominent, abaxial surface dull, whitish, yellowish, greenish or brownish, velutinous to glabrous, primary vein flat or prominent, secondary veins impressed, prominulous or prominent. Inflorescences leaf-opposed, axillary, supra-axillary or terminal, corymbiform dichasia, fascicles or panicles, velutinous to tomentose. Flower buds with corolla lobes twisted or not. Flowers subsessile or pedicellate. Calyx gamosepalous, campanulate, without colleters; lobes 5 or, more rarely, 6-7, subequal, lanceolate or ovate, apex acuminate, acute or obtuse. Corolla gamopetalous, tubular or salverform, white, yellow or orange; lobes erect, patent or deflexed, oblong, filiform, lanceolate, 
ovate or obovate, apex acute or obtuse. Stamens included; anthers free, positioned above the style-head, lanceolate or ovate, apex acuminate, apiculate or acute, base cordate. Ovary superior, hemisyncarpous, 2-carpellate, ovoid or globoid, tomentose to glabrous; style cylindrical; style-head main body oblong or globose, with 2 apical appendages oblong, filiform, ovate or inconspicuous. Follicles 2 or 1 by abortion, flat, falciform, dolabriform, pyriform or suborbicular, smooth, sulcate, verrucose or spinescent, sessile or stipitate, mucronate or not, lenticels conspicuous or inconspicuous, woody, yellow, brown or black, velutinous to glabrous. Seeds oblong, ovate or orbicular, winged, yellow, glabrous; seminal nucleus basal, central, lateral or apical, with or without radial lines.

The information about the protologues of Aspidosperma were checked in their original publications. In the databases "Tropicos" (Missouri Botanical Garden 2015) and "World Checklist of Selected Plant Families" (WCSP 2015), this information is conflicting. The diagnosis of Aspidosperma (A. bicolor Mart. (now a synonym of $A$. pyrifolium), A. macrocarpon Mart., A. pyrifolium Mart., $A$. refractum Mart. (now a synonym of $A$. pyrifolium) and A. tomentosum Mart. (type)), was first published by Martius (1824a) in "Flora", and published again by the same author in "Nova Genera et Species Plantarum" (Martius 1824b, Stafleu \& Cowan 1981, Marcondes-Ferreira 1988). Stafleu \& Cowan (1981) highlighted that Zuccarini should not be cited as co-author of Aspidosperma, since he helped Martius (1824b) only with the organization of the work and did not participate on the original publication (Martius 1824a). This interpretation was later followed by Marcondes-Ferreira (1988) in his study of Aspidosperma and is the interpretation accepted in the present study.

Based on the infrageneric division of Aspidosperma established by Marcondes-Ferreira \& Kinoshita (1996), only species from Aspidosperma subgenus Aspidosperma occur in the state of Pará. Of the nine sections of Aspidosperma subgenus Aspidosperma, seven occur in Pará: Aspidosperma (A. macrocarpon, A. multiflorum A.DC., A. pyrifolium and A. subincanum Mart.), Nobilia (Woodson) Marc.-Ferr. (A. album (Vahl) Benoist ex Pichon, $A$. araracanga Marc.-Ferr., A. desmanthum Benth. ex Müll.Arg., A. eteanum Markgr., A. sandwithianum Markgr. and A. spruceanum Benth. ex Müll.Arg.), Polyneura (Woodson) Marc.-Ferr. (A. cuspa (Kunth) S.F.Blake ex Pittier and A. darienense Woodson ex Dwyer), Inundata Marc.-Ferr. (A. inundatum Ducke), Schultesia Marc.-Ferr. (A. schultesii Woodson), Rigida (Woodson) Marc.Ferr. (A. rigidum Rusby) and Excelsa Marc.-Ferr. (A. carapanauba Pichon, $A$. discolor A.DC., A. excelsum Benth., A. oblongum A.DC. and $A$. salgadense Markgr.) (Marcondes-Ferreira \& Kinoshita 1996).

In Pará, 20 species of Aspidosperma were found, with the Baixo Amazonas Mesoregion presenting the largest number of species (17), and the Metropolitana de Belém Mesoregion presenting the smallest number of species (3) (IBGE 1990). Aspidosperma araracanga and $A$. excelsum are the only species of the genus occurring in all the Mesoregions of Pará (IBGE 1990). We accept Aspidosperma eteanum, A. oblongum, A. salgadense (endemic of Pará) and $A$. sandwithianum as species in this study. Aspidosperma cuspa is a new record for Pará. Furthermore, the fruits of Aspidosperma eteanum and A. salgadense, and the seeds of $A$. eteanum, have been described and illustrated for the first time.

Key to species of Aspidosperma Mart. (Apocynaceae, Rauvolfioideae) in the State of Pará, Northern Brazil.

1.Branches angular ..............................16. A. salgadense

1 '. Branches cylindrical ................................................. 2
2.Branches with cataphylls covering the buds; leaves congested at apex of branches ................................. 3 3.Leaves discolorous; flower buds with corolla lobes not twisted; corolla tubular; ovary tomentose......... 20. A. subincanum

3'.Leaves concolorous; flower buds with corolla lobes twisted; corolla salverform; ovary glabrous ............ 4 4.Leaves membranaceous; flowers 6-6.5 mm long; calyx glabrous externally; corolla lobes filiform.

12. A. multiflorum

4'.Leaves chartaceous; flowers 14-23 mm long; calyx tomentose externally; corolla lobes oblong.

14. A. pyrifolium

2'.Branches without cataphylls covering the buds; leaves arranged along the branches .................................. 5

5. Branches sparsely lenticellate .......................... 6 6.Calyx with 6-7 lobes .............. 5. A. darienense

6'.Calyx with 5 lobes ....................................... 7

7.Venation reticulodromous; inflorescences in panicles 4. A. cuspa

7'.Venation craspedodromous, brochidodromous or eucamptodromous; inflorescences in corymbiform dichasia or fascicles .......................... 8

8.Venation craspedodromous .................. 9

9.Corolla pubescent externally, lobes lanceolate; ovary tomentose; seminal nucleus with radial lines ...........................18. A. schultesii

9'.Corolla glabrous externally, lobes filiform; ovary glabrous; seminal nucleus without radial lines ......................................... 10 10.Corolla tubular, lobes 1-1.5 mm long 8. A. eteanum

10'. Corolla salverform, lobes $2.5-6.5 \mathrm{~mm}$ long ........................................11

11.Leaves with 39-41 pairs of secondary veins, prominent on both surfaces 2. A. araracanga

11'.Leaves with 21-34 pairs of secondary veins, not prominent on both surfaces ......................................12

12.Leaves bullate, adaxial surface dull, with primary vein tomentose 17. A. sandwithianum

12'.Leaves flat, adaxial surface lustrous, with primary vein glabrous

13.Leaves dark green on adaxial surface, with secondary veins prominent; calyx glabrescent to glabrous internally .................... 1. A. album

13'.Leaves olive green or brown on adaxial surface, with secondary veins impressed or prominulous; calyx pubescent at apex internally

14.Leaves with secondary veins impressed on both surfaces ..... 6. A. desmanthum

14'.Leaves with secondary veins prominulous on both surfaces 19. A. spruceanum

8'. Venation brochidodromous or eucamptodromous .......15 
15.Leaves bullate; inflorescences in fascicles; calyx pubescent at base internally .... 3. A. carapanauba

15'.Leaves flat; inflorescences in corymbiform dichasia; calyx pubescent at apex to glabrous internally ...16

16.Leaves concolorous; corolla glabrous externally; follicles not mucronate.

15. A. rigidum

16'.Leaves discolorous; corolla tomentose externally; follicles mucronate ......................... 17

17.Venation eucamptodromous, with $10-13$ pairs of secondary veins ..........11. A. macrocarpon

17'. Venation brochidodromous, with 22-25 pairs of secondary veins

..18

18.Leaves with marginal vein; corolla salverform; anthers lanceolate; ovary tomentose 10. A. inundatum

18 '.Leaves without marginal vein; corolla tubular; anthers ovate; ovary glabrous 9. A. excelsum

5'.Branches densely lenticellate .19

19.Leaves elliptic or ovate, base cuneate or oblique; anthers lanceolate; follicles spinescent........................... 7. A. discolor

19'.Leaves oblong, base revolute; anthers ovate; follicles verrucose ...........13. $A$. oblongum

1. Aspidosperma album (Vahl) Benoist ex Pichon, Bull. Mus. Natl. Hist. Nat. sér. 2 19(4): 367. 1947. (Figure 2a-e).

Trees 15-30 m high; trunk straight. Branches cylindrical, suberous, sparsely lenticellate, pubescent to glabrous, without cataphylls; latex reddish. Leaves alternate, arranged along the branches; petioles 1.2-1.9 cm long, tomentose; blade 7-8.9 x 2.5-3.4 cm, coriaceous, flat, obovate, apex acute, obtuse or retuse, base cuneate or oblique, margin revolute, discolorous, venation craspedodromous, adaxial surface lustrous, dark green, glabrous, primary vein flat, secondary veins prominent, tertiary veins conspicuous, abaxial surface dull, white, tomentose along the primary vein, primary vein prominent, secondary veins prominulous, $22-24$ pairs, tertiary veins conspicuous. Inflorescences $5.7-6.5 \mathrm{~cm}$ long, terminal, corymbiform dichasia, tomentose. Flower buds with corolla lobes twisted. Flowers 5.2-6.5 mm long; pedicellate, pedicel 0.9-1 x 0.5-1 mm, tomentose. Calyx 2-2.5 x 1.5-1.8 mm, tomentose externally, glabrescent to glabrous internally; lobes $5,1.5 \times 1-$ $1.3 \mathrm{~mm}$, ovate, apex acute. Corolla 3.5-5.8 x 1-1.2 mm, salverform, yellow, glabrous externally, pubescent to glabrescent below the anthers internally; lobes $2.5 \times 0.5 \mathrm{~mm}$, erect, filiform, apex acute. Stamens 1-1.5 mm long; filaments 0.5-1 $\mathrm{mm}$ long, pubescent to glabrescent; anthers $0.5 \mathrm{~mm}$ long, ovate, apex apiculate. Carpels 1-1.2 mm long; ovary $0.5 \times 0.5 \mathrm{~mm}$, globoid, glabrous; style 0.3-0.5 mm long; style-head $0.2 \mathrm{~mm}$ long, main body globose, with 2 oblong apical appendages. Follicles 11.7-13 x 7.1-7.3 cm, suborbicular, sulcate, stipitate, mucronate, lenticels inconspicuous, brown, pubescent. Seeds 7.3-9 cm diam., orbicular; seminal nucleus lateral, without radial lines, nucleus $1.8-2 \mathrm{~cm}$ diam.

Material examined: BRAZIL, PARÁ: Almeirim, Monte Dourado, Cova da Onça, 28.VIII.1979, fl., N.T. Silva 5157 (INPA, MG); Almeirim, região do Jarí, Planalto A, km 11, 2.
VIII.1969, fl., N.T. Silva 2569 (IAN, NY-scanned image); Breves, perto do Igarapé Arapijó, transecto para inventário florestal, Q 4-70, 7-30.VII.1956, st., J.M. Pires et al. 5059 (IAN); Breves, transecto para inventário florestal, 20-6 (sp. $\mathrm{n}^{\circ}$ 3), 7-30.VII.1956, fr., J.M. Pires et al. 5290 (IAN); Breves, transecto para inventário florestal, 70-80 (sp. n 7a), 7-30.VII. 1956, fr., J.M. Pires et al. 5454 (IAN); São Sebastião da Boa Vista, Sítio Campina on River Pracuubamirim, 18.X.1984, st., G.L. Sobel et al. 4669A (NY-scanned image).

Additional material: BRAZIL, AMAPÁ: Oiapoque, beira do caminho, 5.X.1949, fl., G.A. Black 49-8406 (IAN); Rio Araguari, on forested island in river, downriver from Porto Platon, 21.IX.1961, fr., J.M. Pires et al. 51149 (IAN, MG); Rio Araguari, upland plant between camps 6 and 7, 12.IX.1961, fl., J.M. Pires et al. 50882 (IAN, RB).

Phytoecological region and phenology: Dense Ombrophilous Forest (terra firme forest). Flowers and fruits collected from August to January.

Common names: araracanga, araracanga-vermelha.

Aspidosperma album occurs in Bolivia, Brazil, Colombia, French Guiana, Guyana, Suriname and Venezuela (Missouri Botanical Garden 2015). In the state of Pará, this species was found in Baixo Amazonas and Marajó Mesoregions (IBGE 1990). In "Livro Vermelho da Flora do Brasil" (Rapini et al. 2013), Aspidosperma album was assigned to the Data Deficient category (DD), but in "Lista de Espécies da Flora e da Fauna Ameaçadas no Estado do Pará" (Pará 2007), it was listed as a Vulnerable species (VU). According to Marcondes-Ferreira \& Kinoshita (1996), Aspidosperma album belongs to section Nobilia. Among the studied species, Aspidosperma album shares morphological features with both $A$. desmanthum and A. spruceanum, especially relating to the flower, but differs from these other two species by the dark green color of the leaf blade and prominent secondary veins on the adaxial surface, and the suborbicular follicles.

2. Aspidosperma araracanga Marc.-Ferr., Revista Brasil. Bot. 14(2): 127. 1991. (Figure 2f-j).

Trees 2.5-44 m high; trunk straight. Branches cylindrical, not suberous, sparsely lenticellate, pubescent to glabrous, without cataphylls; latex orange to reddish. Leaves alternate, arranged along the branches; petioles $1.8-3.5 \mathrm{~cm}$ long, tomentose to glabrous; blade 9.7-10.8 x 3.9-4.8 cm, chartaceous, flat, oblong, elliptic or obovate, apex acuminate, acute or obtuse, base acute, cuneate or oblique, margin revolute, discolorous, venation craspedodromous, adaxial surface lustrous, olive green, glabrous, primary vein prominulous, secondary veins prominent, tertiary veins conspicuous, abaxial surface dull, light green, glabrous, primary vein prominent, secondary veins prominent, 39-41 pairs, tertiary veins conspicuous. Inflorescences $7.7-10.5 \mathrm{~cm}$ long, terminal, corymbiform dichasia, tomentose. Flower buds with corolla lobes twisted. Flowers 8-10.5 mm long; pedicellate, pedicel 1 x $1 \mathrm{~mm}$, tomentose. Calyx 2-2.5 x $1.5 \mathrm{~mm}$, tomentose externally, glabrescent internally; lobes 5, 1-1.5 x $1 \mathrm{~mm}$, ovate, apex acute. Corolla 7-10 × $2 \mathrm{~mm}$, salverform, yellow, glabrous externally, tomentose below the anthers internally; lobes 5.4-6.5 x 0.5 mm, erect, filiform, apex acute. Stamens 2-2.5 mm long; filaments $1.5-2 \mathrm{~mm}$ long, tomentose; anthers $0.5 \mathrm{~mm}$ long, ovate, apex acute. Carpels 2-2.5 mm long; ovary $0.5 \times 0.5 \mathrm{~mm}$, globoid, glabrous; style 1-1.5 mm long; style-head $0.5 \mathrm{~mm}$ long, main body oblong or globose, with 2 oblong apical appendages. Follicles $10.2 \times 8 \mathrm{~cm}$, suborbicular, sulcate, stipitate, mucronate, 
Pereira, A.S.S. et al
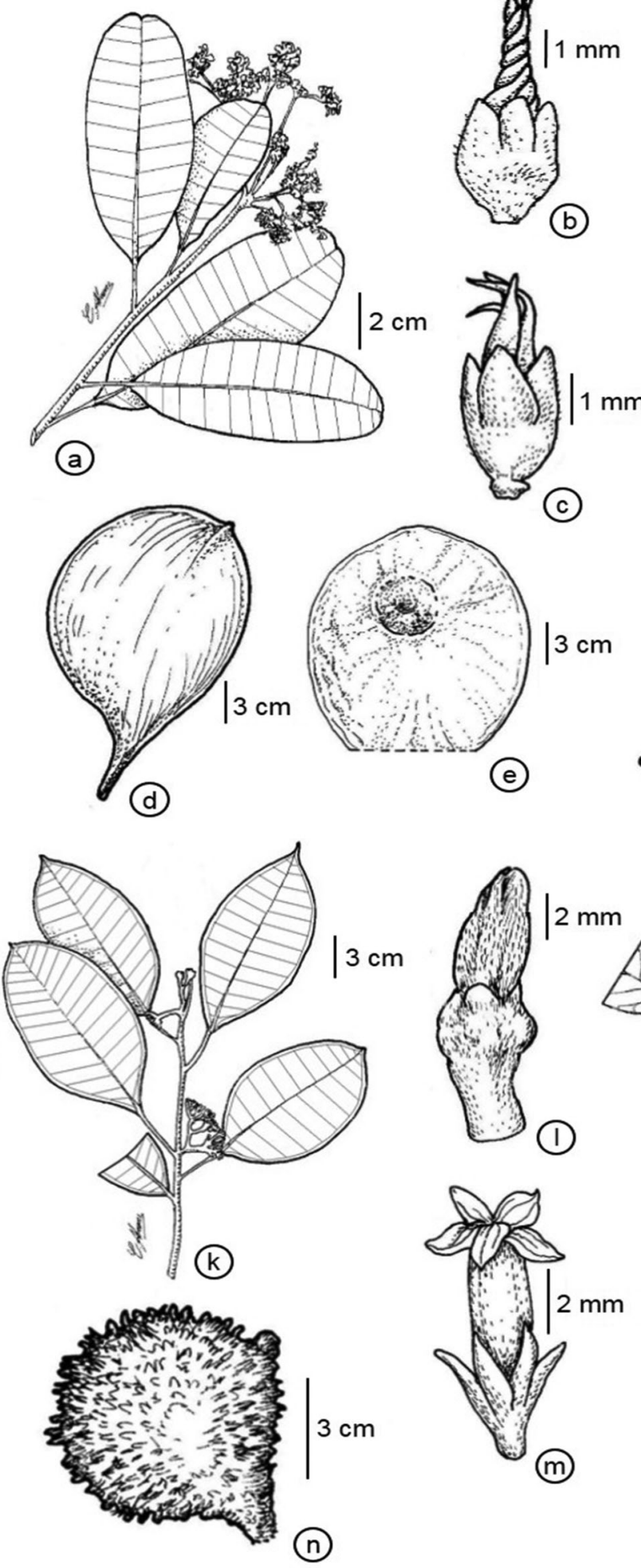
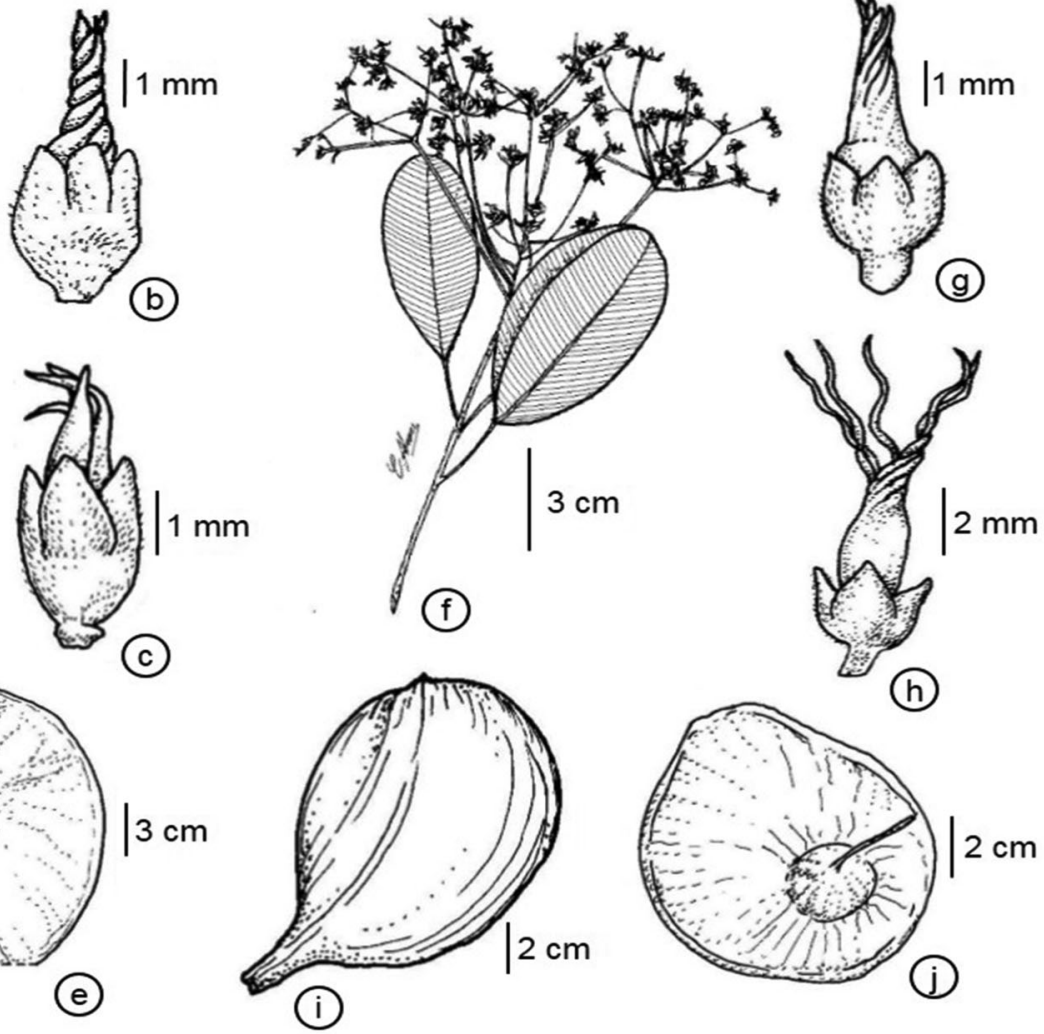
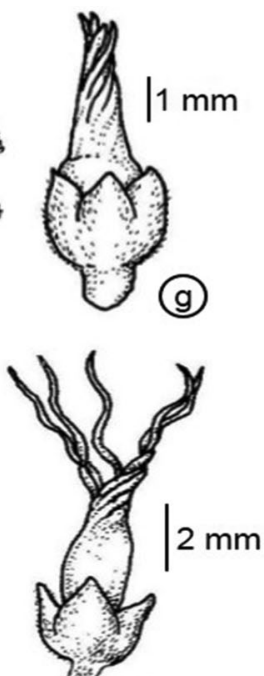

$2 \mathrm{~mm}$<smiles>C1CCCC1</smiles>

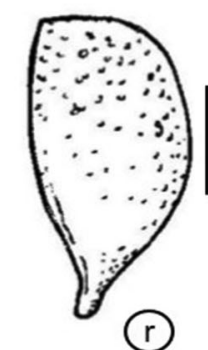

()

Figure 2. a-e. Aspidosperma album. f-j. A. araracanga. k-n. A. carapanauba. o-s. A. cuspa: a, f, k, o. flowering branch; b, g, l, p. flower bud; c, h, m, q. flower; d, i, n, r. follicle; e, j, s. seed. a-e. J.M. Pires et al. 50882; J.M. Pires et al. 51149. f-j. J. Huber 3848; J.M. Pires 11911; R. Romero-Castañeda 1131. k-n. M.G. Silva \& A. Pinheiro 4300; M.G. Silva \& C. Rosário 4847. o-s. G.S. Pinheiro \& J.F.V. Carvalho 674; P.O. Rosa et al. 249.

Figura 2. a-e. Aspidosperma album. f-j. A. araracanga. k-n. A. carapanauba. o-s. A. cuspa: a, f, k, o. ramo florido; b, g, l, p. botão floral; c, h, m, q. flor; d, i, n, r. folículo; e, j, s. semente. a-e. J.M. Pires et al. 50882; J.M. Pires et al. 51149. f-j. J. Huber 3848; J.M. Pires 11911; R. RomeroCastañeda 1131. k-n. M.G. Silva \& A. Pinheiro 4300; M.G. Silva \& C. Rosário 4847. o-s. G.S. Pinheiro \& J.F.V. Carvalho 674; P.O. Rosa et al. 249. 
lenticels inconspicuous, yellow or brown, pubescent. Seeds 7-8 cm diam., orbicular; seminal nucleus lateral, without radial lines, nucleus $2 \mathrm{~cm}$ diam.

Material examined: BRAZIL, PARÁ: Almeirim, Monte Dourado, Perimetral, 23.I.1980, st., N.T. Silva 5420 (MG); Belém, 1.IX.1903, fl., J. Huber 3848 (RB); Belém, IPEAN, Área de Pesquisas Ecológicas do Guamá (APEG), 8.VIII.1968, fl., J.M. Pires 11911 (RB); Belém, IPEAN, Reserva Mocambo, L-12-17, árvore $n^{\circ} 18,8$.VIII.1968, fl., J.M. Pires \& N.T. Silva 11909 (IAN); Bragança, terreno baldio na Rua Edgar Cordeiro de Souza, frente ao portão do Instituto Federal do Pará, 20012002, fl., U. Mehlig 1398 (HBRA); Breves, perto do Igarapé Arapijó, transecto para inventário florestal, Q 1-76, 7-30. VII.1976, st., J.M. Pires et al. 5083 (IAN); Cachoeira do Arari, Rio Camará, Fazenda Gurupatuba, 8.VIII.1950, fl., G.A. Black 50-9924 (NY-scanned image); Colares, 18.VIII.1913, fl., A. Ducke s.n. (RB 13333); Maracanã, área do centro de treinamento, 4.VII.1977, fl., E. Oliveira 6650 (MG, NYscanned image); Melgaço, Caxiuanã, Rio Caxiuanã, pt. 02, 9.IX.1983, st., B. Pena 1027 (INPA); Oriximiná, Baixo Trombetas, 14.IX.1910, fl., A. Ducke s.n. (RB 13332); Oriximiná, Lago Salgado (Rio Trombetas), 25.IV.1917, fr., A. Ducke s.n. (RB 22441); Santarém, Reserva Curuá-Una, Parque Fenológico, árvore $\mathrm{n}^{\circ}$ 152, 19.IV.1999, st., M.R. Cordeiro 3811 (IAN); São Sebastião da Boa Vista, Sítio Campina on River Pracuubamirim, 17.X.1984, st., G.L. Sobel et al. 4638 (NY-scanned image); Tucuruí, Transgoiânia, área nuclear $n^{\circ}$ 4, III.1981, st., P. Lisboa et al. 3017 (MG); Vitória do Xingu, Usina Hidrelétrica Belo Monte, 29.V.2012, f1., L.C. Antônio PSACF 276 (MG).

Additional material: BRAZIL, AMAZONAS: Parintins, Lago José-Assú, 18.IX.1932, fl., A. Ducke s.n. (RB 24572); MATO GROSSO: Gaúcha do Norte, Fazenda Pontal, propriedade de Silvino Perotto, 16.VIII.2000, fl., N.M. Ivanauskas 4354 (UEC); RONDÔNIA: Vicinity of Santa Bárbara, $15 \mathrm{~km}$ east of $\mathrm{km} \mathrm{117,} \mathrm{Porto} \mathrm{Velho} \mathrm{to} \mathrm{Cuiabá} \mathrm{highway,}$ 16.VIII.1968, fl., G.T. Prance \& J.F. Ramos 7016 (MG). COLOMBIA, BOLÍVAR: Palotal, 4.VII.1948, fr., R. RomeroCastañeda 1131 (COL-scanned image).

Phytoecological region and phenology: Dense Ombrophilous Forest (terra firme forest). Flowers and fruits collected from June to April.

Common names: araracanga, aracandeua.

Aspidosperma araracanga occurs in Brazil, Colombia, Peru and Venezuela (Marcondes-Ferreira 1991, Missouri Botanical Garden 2015). In the state of Pará, this species was found in all Mesoregions (IBGE 1990). According to Marcondes-Ferreira \& Kinoshita (1996), Aspidosperma araracanga belongs to section Nobilia. Aspidosperma araracanga resembles A. desmanthum, especially flower features, but is distinguished from A. desmanthum, and from the other species in section Nobilia occurring in Pará, by the number of secondary veins (39-41 pairs), with the veins being prominent on both leaf surfaces.

3. Aspidosperma carapanauba Pichon, Bull. Mus. Natl. Hist. Nat. sér. 2 19(4): 365. 1947. (Figure 2k-n).

Trees 20-38 m high; trunk tortuous. Branches cylindrical, suberous, sparsely lenticellate, tomentose to pubescent, without cataphylls; latex whitish. Leaves alternate, arranged along the branches; petioles $2.4-2.9 \mathrm{~cm}$ long, tomentose to pubescent; blade 9.4-17.1 x 4.1-7.8 cm, subcoriaceous to coriaceous, bullate, oblong, elliptic or ovate, apex acuminate or acute, base cuneate or oblique, margin revolute, discolorous, venation brochidodromous, with marginal vein $2-4 \mathrm{~mm}$ from the margin, adaxial surface dull or lustrous, brown or black, pubescent along the primary vein, primary vein flat, secondary veins impressed, tertiary veins conspicuous, abaxial surface dull, yellow, velutinous, primary vein prominent, secondary veins prominent, 13-22 pairs, tertiary veins inconspicuous. Inflorescences $2.4-4.8 \mathrm{~cm}$ long, axillary or supraaxillary, fascicled, tomentose. Flower buds with corolla lobes not twisted. Flowers 12.5-13.5 mm long; pedicellate, pedicel 3.5-4 x 1.5-2 mm, tomentose. Calyx 3-3.5 x $3.5 \mathrm{~mm}$, tomentose externally, pubescent at base internally; lobes 5, 2-3 x 1.2-2 mm, ovate, apex acute. Corolla 6-7.5 x 2.5-3 mm, tubular, white, tomentose externally, pubescent to glabrescent along the tube internally; lobes 1.5-2.5 x $1 \mathrm{~mm}$, erect, ovate, apex acute. Stamens 2.5-4 mm long; filaments 1.7-3 mm long, pubescent to glabrescent; anthers 0.8-1 $\mathrm{mm}$ long, ovate, apex acute. Carpels $2 \mathrm{~mm}$ long; ovary 1 x $1.5 \mathrm{~mm}$, globoid, tomentose; style $0.8 \mathrm{~mm}$ long; style-head $0.2 \mathrm{~mm}$ long, main body oblong, with 2 inconspicuous apical appendages. Follicles 5.5-6.5 x 4.6-5 cm, dolabriform, spinescent, sessile or stipitate, mucronate, lenticels inconspicuous, brown, pubescent. Seeds not observed.

Material examined: BRAZIL, PARÁ: Almeirim, região do Jarí, estrada do Munguba, km 12, 11.VIII.1969, fl., N.T. Silva 2642 (IAN); Almeirim, região do Jarí, Monte Dourado, serra de 1,40 m de altura, a $3 \mathrm{~km}$ da margem, 3.XII.1967, fl., E. Oliveira 3799 (IAN); Almeirim, região do Rio Jarí, Monte Dourado, Planalto A, 9.IX.1968, fl., N.T. Silva 912 (IAN); Almeirim, região do Jarí, Planalto Monte Dourado, 22.I.1968, fl., E. Oliveira 3945 (IAN); Almeirim, região do Rio Jarí, Monte Dourado, Planalto, 2.II.1968, fr., E. Oliveira 4076 (IAN).

Additional material: BRAZIL, AMAPÁ: Mazagão, área do Felipe 03, $0^{\circ} 40^{\prime} \mathrm{S}, 52^{\circ} 18^{\prime} \mathrm{W}, 3 . V I .1983$, fl., N.T. Silva 5296 (MG); MATO GROSSO: Aripuanã, km 238 da BR-174, núcleo Juina, área urbana, 17.I.1979, fr., M.G. Silva \& A. Pinheiro 4300 (MG, RB, UEC); Guarantã, adjacências do Hotel Floresta Amazônica, 7.VI.1997, fl., G.F. Árbocz et al. 4000 (UEC); km 330 da rod. BR-174, margem da Fazenda Vale do Tucanã, 10.VI.1979, fl., M.G. Silva \& C. Rosário 4847 (MG, RB).

Phytoecological region and phenology: Dense Ombrophilous Forest (terra firme forest). Flowers and fruits collected from August to February.

Common names: carapanaúba, carapanaúba-preta.

According to Koch et al. (2015), Aspidosperma carapanauba is a species endemic to Brazil, and occurs in the states of Amapá, Amazonas, Mato Grosso, Pará and Rondônia. In the state of Pará, this species was found only in Baixo Amazonas Mesoregion (IBGE 1990). As stated by the classification from Marcondes-Ferreira \& Kinoshita (1996), it belongs to section Excelsa. Although seeds were not observed here, MarcondesFerreira (1988) described them as nearly orbicular (ca. $4.5 \mathrm{~cm}$ diam.) with a lateral seminal nucleus (ca. $2.5 \mathrm{~cm}$ diam.). Aspidosperma carapanauba was one of the most easily recognized species, and is distinguished from the other species in section Excelsa occurring in Pará by the larger petioles (2.4-2.9 cm long) and leaf blades (9.4-17.1 x 4.1-7.8 cm), bullate leaves, fasciculate inflorescences and larger flowers (12.5-13.5 mm long).

4. Aspidosperma cuspa (Kunth) S.F.Blake ex Pittier, Man. Pl. Usual. Venez.: 110. 1926. (Figure 2o-s).

Trees 4-27 m high; trunk straight. Branches cylindrical, not suberous, sparsely lenticellate, glabrescent to glabrous, without cataphylls; latex whitish. Leaves alternate, arranged along the branches; petioles $0.4-0.6 \mathrm{~cm}$ long, pubescent to glabrescent; 
blade 2.7-6 x 1.4-2.6 cm, chartaceous, flat, oblong or obovate, apex acute or obtuse, base cuneate, attenuate or oblique, margin revolute, discolorous, venation reticulodromous, adaxial surface dull, olive green, glabrous, primary vein prominulous, secondary veins prominent, tertiary veins conspicuous, abaxial surface dull, light green, pubescent, primary vein prominent, secondary veins prominulous, 9-12 pairs, tertiary veins inconspicuous. Inflorescences $1.5-11.3 \mathrm{~cm}$ long, leafopposed or axillary, panicled, tomentose. Flower buds with corolla lobes not twisted. Flowers 4.5-5 mm long; subsessile or pedicellate, pedicel 1 x $0.5 \mathrm{~mm}$, glabrous. Calyx 1.3-1.5 x $1.2 \mathrm{~mm}$, glabrous on both surfaces, except at margin; lobes $5,1 \mathrm{x}$ 0.5-0.7 mm, ovate, apex acute. Corolla 3-3.5 x $1.2 \mathrm{~mm}$, tubular, yellow, glabrous externally, tomentose below the anthers internally; lobes $1-1.3$ x $0.6 \mathrm{~mm}$, erect, oblong, apex obtuse. Stamens 2.2-2.7 mm long; filaments $1.5-2 \mathrm{~mm}$ long, tomentose; anthers 0.7 $\mathrm{mm}$ long, lanceolate, apex acute. Carpels $1.6 \mathrm{~mm}$ long; ovary $0.7 \mathrm{x}$ $0.5 \mathrm{~mm}$, ovoid, glabrous; style $0.5 \mathrm{~mm}$ long; style-head $0.4 \mathrm{~mm}$ long, main body globose, with 2 oblong apical appendages. Follicles 2.5-3 x 1.3-1.5 cm, dolabriform, smooth, stipitate, mucronate, lenticels conspicuous, yellow, pubescent. Seeds 2.2$2.4 \times 0.9-1 \mathrm{~cm}$, oblong or ovate; seminal nucleus basal or apical, without radial lines, nucleus $1.1 \times 0.8 \mathrm{~cm}$.

Material examined: BRAZIL, PARÁ: Marabá, EstreitoMarabá, km 2, 9.IV.1974, fr., G.S. Pinheiro \& J.F.V. Carvalho 674 (IAN).

Additional material: BRAZIL, GOIÁS: $12^{\circ} 15^{\prime} \mathrm{S}, 46^{\circ} 45^{\prime} \mathrm{W}$, 21.VI.1979, fr., L.C.O. Filho 29 (RB); MARANHÃO: São Francisco do Maranhão, 21.V.2009, fr., L. Rodrigues et al. s.n. (UFRN 9480-scanned image); MATO GROSSO: Barra do Garças, Fazenda Taquaral, Cabeceira do Rio Pindaíba, 25. X.2003, fl., C. Fernandes-Bulhão et al. 358CFB (UEC); Barra do Garças, Serra do Taquaral, mata em grotão, 25.XI.1997, fl., L.C. Bernacci \& G.F. Árbocz 2616 (IAC, UEC); Santo Antônio de Leverger, 25.III.1982, fr., A.L. Prado et al. 327 (UEC); MINAS GERAIS: Araguari, Funil I, Capim Branco I, 15.XII.2006, fl., P. O. Rosa et al. 249 (UEC).

Phytoecological region and phenology: Savannah. Flowers and fruits collected from April to September.

Common names: guatambuzinho (Koch et al. 2015).

According to Marcondes-Ferreira (1988), Aspidosperma cuspa has the widest distribution of the genus, occurring from Haiti to Paraguay, in many plant formations. Aspidosperma cuspa is a new record for the state of Pará, being found only in Sudeste Paraense Mesoregion (IBGE 1990). In the classification from Marcondes-Ferreira \& Kinoshita (1996), Aspidosperma cuspa belongs to section Polyneura. It can be easily differentiated from the other species that occur in Pará by the reticulodromous venation, paniculate inflorescence and smaller seeds (2.2-2.4 cm long) with basal or apical seminal nucleus.

5. Aspidosperma darienense Woodson ex Dwyer, Ann. Missouri Bot. Gard. 53(1): 104. 1966. (Figure 3a-d).

Trees 10-38 m high; trunk tortuous. Branches cylindrical, not suberous, sparsely lenticellate, pubescent, without cataphylls; latex whitish. Leaves alternate, arranged along the branches; petioles $0.7-1.1 \mathrm{~cm}$ long, tomentose to pubescent; blade $8.9-21 \mathrm{x}$ $3.5-7.8 \mathrm{~cm}$, coriaceous, flat, oblong or obovate, apex acuminate, acute or obtuse, base cuneate, margin revolute, discolorous, venation brochidodromous, with marginal vein $1 \mathrm{~mm}$ from the margin, adaxial surface lustrous, dark brown, glabrous, primary vein prominulous, secondary veins impressed, tertiary veins conspicuous, abaxial surface dull, light brown, pubescent, primary vein prominent, secondary veins impressed, 30-38 pairs, tertiary veins inconspicuous. Inflorescences $3.2-4 \mathrm{~cm}$ long, supraaxillary, corymbiform dichasia, tomentose. Flower buds with corolla lobes not twisted. Flowers 6.5-8.5 mm long; subsessile or pedicellate, pedicel $1-2.5 \times 1 \mathrm{~mm}$, tomentose. Calyx $2.3-2.5 \mathrm{x}$ 2-2.5 $\mathrm{mm}$, tomentose to pubescent externally, glabrous internally; lobes 6-7, 1.5-2 x 1.5-2 mm, ovate, apex obtuse. Corolla 4.5-6 x $1.5 \mathrm{~mm}$, salverform, white, tomentose externally, tomentose along the corolla internally; lobes $2.5-3 \times 1 \mathrm{~mm}$, erect, lanceolate, apex acute or obtuse. Stamens 1.5-2 mm long; filaments $0.5-1 \mathrm{~mm}$ long, tomentose; anthers $1 \mathrm{~mm}$ long, lanceolate, apex acuminate. Carpels $1.7 \mathrm{~mm}$ long; ovary $0.7 \mathrm{x}$ $0.8 \mathrm{~mm}$, globoid, pubescent at apex; style $0.8 \mathrm{~mm}$ long; stylehead $0.2 \mathrm{~mm}$ long, main body oblong, with 2 inconspicuous apical appendages. Follicles 7.4-10.2 x 4.4-4.6 cm, falciform or dolabriform, smooth, sessile, mucronate, lenticels conspicuous, brown, glabrous. Seeds 6.7-7 x 4.3-5.2 cm, ovate; seminal nucleus central, without radial lines, nucleus $2.9 \mathrm{~cm}$ diam.

Material examined: BRAZIL, PARÁ: Almeirim, Monte Dourado, próximo a marina da SION em Munguba, 26.IX. 1986, fr., M.J. Pires \& N.T. Silva 1390 (INPA, MG); Almeirim, Monte Dourado, Bom Futuro, 10.III.1981, fr., N.T. Silva 5273 (INPA, MG); Almeirim, região do Rio Jarí, Monte Dourado, Planalto B, entre Pilão e Repartimento, 28.X.1968, fl., N.T. Silva 1325 (IAN, NY-scanned image).

Additional material: BRAZIL, AMAPÁ: Rio Araguari, upriver, 20 minutes from camp 5 toward camp 4, 8.IX.1961, fr., J.M. Pires et al. 50756 (BHCB, IAN, MG); Rio Araguari, vic. camp 12, 28.IX.1961, fl., J.M. Pires et al. 51291 (IAN, MG); Rio Oiapoque, about $0.5 \mathrm{~km}$ south of mouth of Rio Muturá, 21. IX.1960, fl., H.S. Irwin et al. 48412 (BHCB, MG). COLOMBIA, NORTE DE SANTANDER: San José de Cúcuta, Aguaclara, Hacienda San Roque, 26.V.1970, fr., R.J. Mejía 4913 (COLscanned image). ECUADOR, PASTAZA: Pozo petrolero Villano 2 de ARCO, 1-18.XII.1991, fl., F. Hurtado 2868 (UEC).

Phytoecological region and phenology: Dense Ombrophilous Forest (terra firme and várzea forests). Flowers and fruits collected from September to March.

Common names: araracanga-da-várzea, araracanga, garlipo. Aspidosperma darienense occurs in Brazil, Colombia, Ecuador, French Guiana, Panama and Suriname (Missouri Botanical Garden 2015). In the state of Pará, this species was found only in Baixo Amazonas Mesoregion (IBGE 1990). In "The IUCN Red List of Threatened Species 2015.2" (IUCN 2015), Aspidosperma darienense was assigned to the Endangered category (EN). According to Marcondes-Ferreira \& Kinoshita (1996), Aspidosperma darienense belongs to section Polyneura. This species is easily distinguished from the other species analysed by the calyx, which has 6-7 lobes. In fact, according to Marcondes-Ferreira (1988) this feature distinguishes Aspidosperma dariense from all other species in the genus, as well as almost all other species from Apocynaceae.

6. Aspidosperma desmanthum Benth. ex Müll.Arg. in C.F.P. von Martius \& auct. suc. (eds.), Fl. Bras. 6(1): 51. 1860. (Figure 3e-i).

Trees 6-35 m high; trunk straight. Branches cylindrical, suberous, sparsely lenticellate, pubescent to glabrescent, without cataphylls; latex reddish. Leaves alternate, arranged along the branches; petioles $0.6-2.1 \mathrm{~cm}$ long, pubescent to glabrescent; blade 7.2-11.5 x 2.7-5.5 cm, coriaceous, flat, elliptic or obovate, apex acute, retuse or emarginate, base cuneate or oblique, margin straight or revolute, discolorous, venation craspedodromous, 

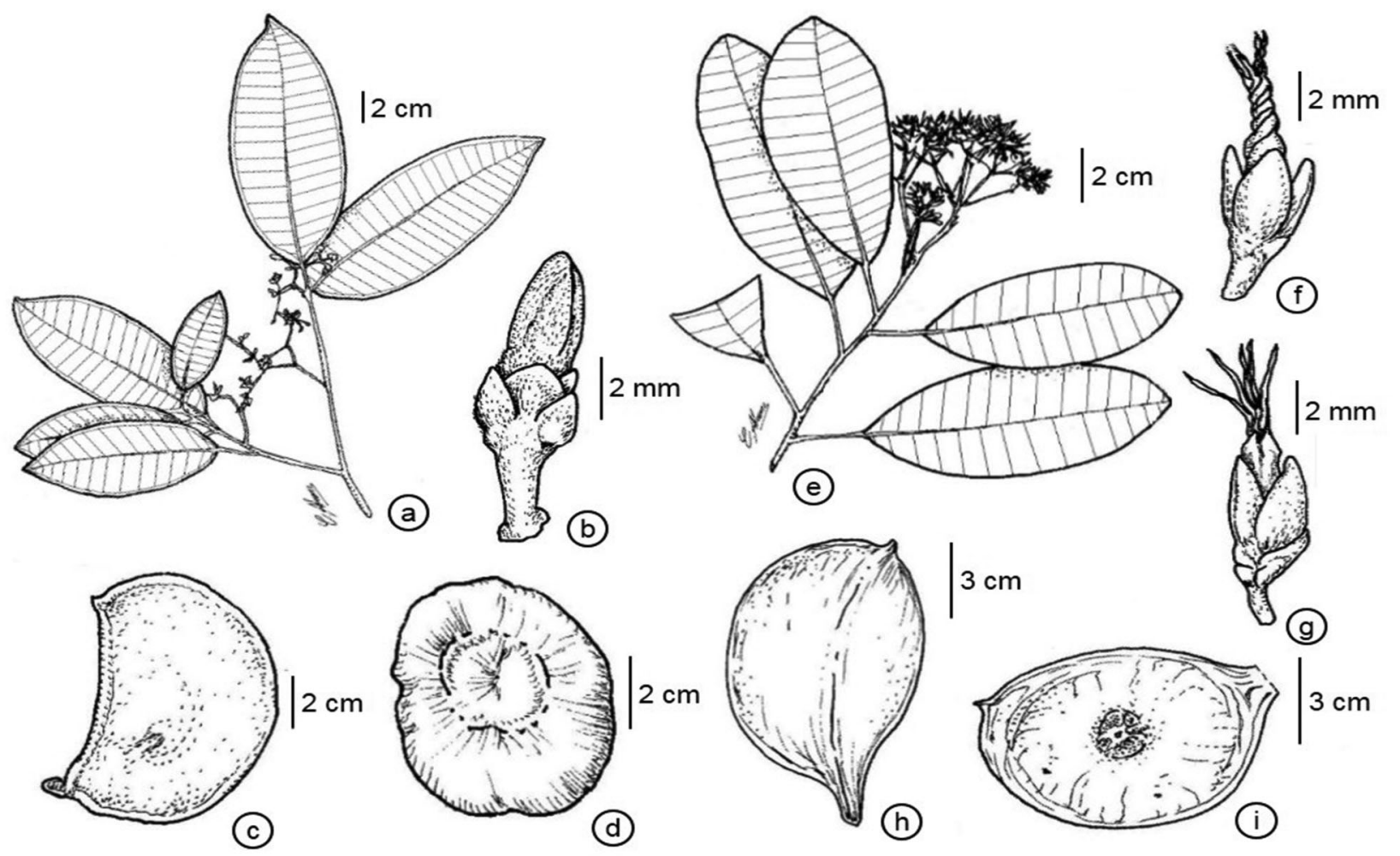

$3 \mathrm{~cm}$
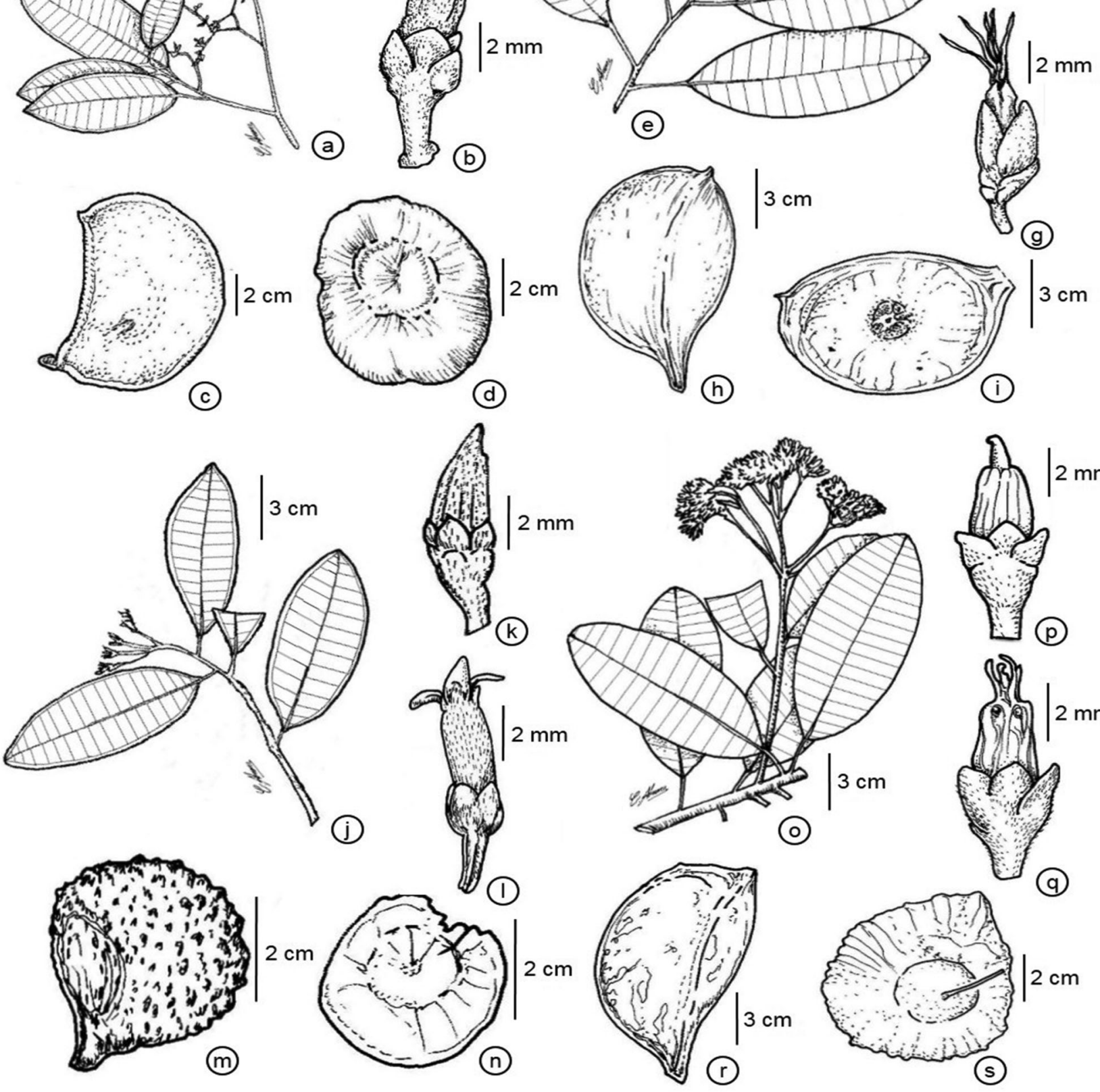

Figure 3. a-d. Aspidosperma darienense. e-i. A. desmanthum. j-n. A. discolor. o-s. A. eteanum: a, e, j, o. flowering branch; b, f, k, p. flower bud; g, l, q. flower; c, h, m, r. follicle; d, i, n, s. seed. a-d. N.T. Silva 1325; M.J. Pires \& N.T. Silva 1390; R.J. Mejía 4913. e-i. M. Pacheco et al. 164; R. Souza 10348. j-n. A.P. Duarte 8179; H.S. Irwin et al. 17160; R.L. Fróes 33480. o-s. A. Ducke s.n. (RB 22445); E. Oliveira 4033.

Figura 3. a-d. Aspidosperma darienense. e-i. A. desmanthum. j-n. A. discolor. o-s. A. eteanum: a, e, j, o. ramo florido; b, f, k, p. botão floral; g, l, q. flor; c, h, m, r. folículo; d, i, n, s. semente. a-d. N.T. Silva 1325; M.J. Pires \& N.T. Silva 1390; R.J. Mejía 4913. e-i. M. Pacheco et al. 164; R. Souza 10348. j-n. A.P. Duarte 8179; H.S. Irwin et al. 17160; R.L. Fróes 33480. o-s. A. Ducke s.n. (RB 22445); E. Oliveira 4033. 
adaxial surface lustrous, olive green or brown, glabrous, primary vein flat, secondary veins impressed, tertiary veins inconspicuous, abaxial surface dull, light green, glabrous, primary vein prominent, secondary veins impressed, 21-26 pairs, tertiary veins inconspicuous. Inflorescences $6.1-10.3 \mathrm{~cm}$ long, terminal, corymbiform dichasia, tomentose. Flower buds with corolla lobes twisted. Flowers 5-11 mm long; pedicellate, pedicel 0.2-2.3 x 0.5$1 \mathrm{~mm}$, tomentose. Calyx 2-3.5 x 2-3 mm, tomentose externally, pubescent at apex internally; lobes 5, 2-2.5 x 1-1.2 mm, lanceolate or ovate, apex acute. Corolla 3.5-8 x 1.5-2 mm, salverform, yellow, glabrous externally, tomentose below the anthers internally; lobes 2.5-5 x $0.3 \mathrm{~mm}$, erect, filiform, apex acute. Stamens $1.5-2.5 \mathrm{~mm}$ long; filaments 1-2 mm long, tomentose; anthers $0.5 \mathrm{~mm}$ long, ovate, apex acute. Carpels $1.5 \mathrm{~mm}$ long; ovary 0.5 x $0.7 \mathrm{~mm}$, globoid, glabrous; style $0.7 \mathrm{~mm}$ long; style-head $0.3 \mathrm{~mm}$ long, main body oblong, with 2 oblong apical appendages. Follicles 10.4-10.6 x 6.8-7.2 cm, dolabriform, sulcate, stipitate, mucronate, lenticels inconspicuous, yellow, pubescent to glabrescent. Seeds 6.9-7.3 cm diam., orbicular; seminal nucleus central, without radial lines, nucleus $1.5-2 \mathrm{~cm}$ diam.

Material examined: BRAZIL, PARÁ: Itaituba, Rio Tapajós, 1.IX.1916, fl., A. Ducke s.n. (RB 22439); Itaituba, Rio Tapajós, Pimental, 5.II.1917, st., A. Ducke s.n. (RB 22442); Itaituba, Rio Tapajós próximo a boca do Rio Rato, margem direita do Rio Tapajós, s.d., fr., I.L. do Amaral et al. 3738 (INPA); Jacareacanga, Parque Nacional do Tapajós, Ilha do Pacú no Tapajós, 30.XI.1978, fr., M.G. Silva \& C. Rosário 4031 (MG, NY-scanned image).

Additional material: BRAZIL, ACRE: Cruzeiro do Sul, nos arredores do acampamento do projeto RADAM, próximo do aeroporto novo, 11.II.1976, st., D.P. Monteiro \& C. Damião 321 (MG); AMAZONAS: Manaus, XI.1942, fr., A. Ducke 1156 (MG); Manaus, Distrito Agropecuário, Fazenda Porto Alegre, reserva 3402 (Cabo Frio) of the WWF/INPA MCS project, $02^{\circ} 25^{\prime} 25^{\prime}$ 'S, 59 $54^{\prime} 38^{\prime \prime W}, 28 . I .1989$, fl., M. Pacheco et al. 164 (NY-scanned image); Manaus, Reserva Florestal Ducke, Manaus-Itacoatiara, km 26, 23.IV.1995, fl., P. A.C.L. Assunção \& E.C. Pereira 190 (MG, RB); MATO GROSSO: Aripuanã, Rio Juruena, Fontanilha, arredores do aeroporto, 18.VII.1977, f1., M.G. Silva 3375 (MG); Expedition base camp, $12^{\circ} 49^{\prime} \mathrm{S}, 51^{\circ} 46^{\prime} \mathrm{W}$, just north of base camp, 29. IX.1968, fr., R. Souza 10348 (RB); RONDÔNIA: Basin of Rio Madeira, Rio Laje on road Guajará-Mirim to Ribeirão, 3. VIII.1968, fl., G.T. Prance et al. 6741 (MG).

Phytoecological region and phenology: Dense Ombrophilous Forest (terra firme forest). Flowers and fruits collected from September to November.

Common names: araracanga, piquiá-marfim-do-roxo, quinada-mata.

Aspidosperma desmanthum occurs in Belize, Bolivia, Brazil, Costa Rica, Guatemala, Mexico, Nicaragua and Panama (Missouri Botanical Garden 2015). In the state of Pará, this species was found only in Sudoeste Paraense Mesoregion (IBGE 1990). In "Lista de Espécies da Flora e da Fauna Ameaçadas no Estado do Pará" (Pará 2007), Aspidosperma desmanthum was listed as a Vulnerable species (VU). According to Marcondes-Ferreira \& Kinoshita (1996), it belongs to section Nobilia. Among the studied species, Aspidosperma desmanthum was one of the most difficult to distinguish. This species shares morphological features with both Aspidosperma album and A. araracanga, mainly flower structures, but differs from them by the impressed secondary veins on both leaf surfaces and dolabriform follicle. Aspidosperma desmanthum also shares morphological features with $A$. eteanum, especially leaf traits, but differs from $A$. eteanum by the impressed secondary veins on the abaxial surface, salverform corolla, length of corolla lobes (2.5-5 mm long) and orbicular seeds. Aspidosperma desmanthum is most often confused with A. spruceanum, which was highlighted by Marcondes-Ferreira (1988), but can be distinguished from $A$. spruceanum by the impressed secondary veins on both leaf surfaces.

7. Aspidosperma discolor A.DC. in A.P.de Candolle, Prodr. 8: 398. 1844. (Figure 3j-n).

Trees 6-28 m high; trunk tortuous. Branches cylindrical, not suberous, densely lenticellate, pubescent to glabrescent, without cataphylls; latex whitish. Leaves alternate, arranged along the branches; petioles 1-1.2 cm long, pubescent; blade 6.6-8.3 x 2-3.5 $\mathrm{cm}$, chartaceous to subcoriaceous, flat, elliptic or ovate, apex acuminate or acute, base cuneate or oblique, margin revolute, discolorous, venation brochidodromous, with marginal vein $1 \mathrm{~mm}$ from the margin, adaxial surface lustrous, olive green, glabrous, primary vein flat, secondary veins prominent, tertiary veins inconspicuous, abaxial surface dull, light green, pubescent, primary vein prominent, secondary veins prominent, 16-22 pairs, tertiary veins inconspicuous. Inflorescences $3.5-4.5 \mathrm{~cm}$ long, terminal, corymbiform dichasia, tomentose. Flower buds with corolla lobes not twisted. Flowers $6.5-8.5 \mathrm{~mm}$ long; pedicellate, pedicel 1.7-3 x $0.7 \mathrm{~mm}$, tomentose. Calyx 2-2.5 x 1-2 mm, tomentose externally, pubescent to glabrous at apex internally; lobes 5, 1-1.5 x 1-2 mm, ovate, apex acute or obtuse. Corolla 6.5-7 $x$ 1.5-2 mm, tubular, white, tomentose externally, pubescent below the anthers internally; lobes $2-3.5 \times 0.3 \mathrm{~mm}$, patent, ovate, apex acute. Stamens 3-5 mm long; filaments 2-4 mm long, pubescent; anthers $1 \mathrm{~mm}$ long, lanceolate, apex acute. Carpels 1.3-2.8 mm long; ovary 0.7-1 x 1-1.2 mm, globoid, tomentose; style 0.3-1.5 $\mathrm{mm}$ long; style-head $0.3 \mathrm{~mm}$ long, main body oblong, with 2 oblong apical appendages. Follicles 4-4.5 x 3.5-3.7 cm, dolabriform or suborbicular, spinescent, stipitate, mucronate, lenticels inconspicuous, brown, pubescent. Seeds 3-3.5 cm diam., orbicular; seminal nucleus central, without radial lines, nucleus $1.7 \mathrm{~cm}$ diam.

Material examined: BRAZIL, PARÁ: Canaã dos Carajás, área da Mina do Sossego, 25.XI.2009, fl., R.D. Ribeiro et al. 1387 (MG, RB).

Additional material: BRAZIL, GOIÁS: Goiânia, Alexânia, 13.VII.1964, fr., A.P. Duarte 8179 (RB); Luziânia, Fazenda Suindara do Alagado, ponto 14, 08.XI.2002, fl., G. PereiraSilva et al. 6967 (UEC); MARANHÃO: Santa Quitéria, Fazenda Marflora, 7.IX.1993, fr., B.A.S. Pereira 2519 (UEC); MATO GROSSO: Barra do Garças, Serra do Taquaral, mata em grotão, 24.XI.1997, fl., L.C. Bernacci \& G.F. Árbocz 2593 (IAC, UEC); Drainage of the upper Rio Araguaia, lower slopes, Serra Azul, ca. $85 \mathrm{~km} \mathrm{~S}$ of Xavantina, 15.VI.1966, fr., H.S. Irwin et al. 17160 (NY-scanned image, RB); MINAS GERAIS: Presidente Olegário, região do Vale do Rio Paranaíba, próximo das Cabeceiras do Rio São Francisco, 7.IX.1957, fl., R.L. Fróes 33480 (IAN).

Phytoecological region and phenology: Savannah. Flowers and fruits collected from November to June.

Common names: carapanaúba, peroba, peroba de gomo.

Aspidosperma discolor occurs in Brazil, Suriname and Venezuela (Missouri Botanical Garden 2015). In the state of Pará, this species was found only in Sudeste Paraense Mesoregion (IBGE 1990). According to Marcondes-Ferreira \& Kinoshita (1996), Aspidosperma discolor belongs to section 
Excelsa. It shares morphological features with both Aspidosperma oblongum and A. salgadense, mainly flower structures, but differs from the former by the elliptic or ovate leaf blades with cuneate or oblique base, lanceolate anthers and spinescent follicles, and from the latter by the cylindrical branches and tomentose ovary.

8. Aspidosperma eteanum Markgr., Notizbl. Bot. Gart. Berlin-Dahlem 12: 297. 1935. (Figure 3o-s).

Trees 12-38 m high; trunk straight. Branches cylindrical, suberous or not, sparsely lenticellate, pubescent, without cataphylls; latex orange to reddish. Leaves alternate, arranged along the branches; petioles $1-2 \mathrm{~cm}$ long, pubescent; blade 7.5-12.2 x 2.9-5 cm, coriaceous, flat, oblong or ovate, apex acuminate or acute, base cuneate or oblique, margin straight or revolute, discolorous, venation craspedodromous, adaxial surface lustrous, dark brown, glabrous, primary vein flat, secondary veins impressed, tertiary veins inconspicuous, abaxial surface dull, light brown, glabrous, primary vein prominent, secondary veins prominulous, 24-26 pairs, tertiary veins inconspicuous. Inflorescences $6.2-7.8 \mathrm{~cm}$ long, axillary or terminal, corymbiform dichasia, tomentose. Flower buds with corolla lobes twisted. Flowers 8-9 mm long; pedicellate, pedicel 1.5-3 x 0.7-1 mm, tomentose. Calyx 2.5-3 x 2.5-3 mm, tomentose externally, pubescent at apex internally; lobes 5, 1.5-2.5 x 1.7-2 $\mathrm{mm}$, ovate, apex acute. Corolla 5-6 x $2 \mathrm{~mm}$, tubular, yellow, glabrous externally, tomentose below the anthers internally; lobes $1-1.5$ x $0.3 \mathrm{~mm}$, erect, filiform, apex acute. Stamens 2-3 mm long; filaments 1-2 mm long, tomentose; anthers $1 \mathrm{~mm}$ long, ovate, apex acuminate or acute. Carpels $1.5 \mathrm{~mm}$ long; ovary $0.6 \mathrm{x}$ $0.7 \mathrm{~mm}$, globoid, glabrous; style $0.6 \mathrm{~mm}$ long; style-head $0.3 \mathrm{~mm}$ long, main body oblong, with 2 oblong apical appendages. Follicles $8-9.5$ x $6.5 \mathrm{~cm}$, dolabriform, sulcate, stipitate, mucronate, lenticels inconspicuous, yellow or brown, pubescent. Seeds $6.5 \times 6 \mathrm{~cm}$, ovate; seminal nucleus lateral, without radial lines, nucleus $2.5 \mathrm{~cm}$ diam.

Material examined: BRAZIL, PARÁ: Almeirim, Monte Dourado, 28.I.1968, fr., E. Oliveira 4033 (IAN); Almeirim, Serra de Almeirim, 24.VIII.1918, fl., A. Ducke s.n. (RB 22445); Altamira, margem direita do Rio Ariri (serraria Banach), 28. VIII.1986, fl., A.T.G. Dias et al. 103 (MG); Aveiro, região do Tapajós, Boa Vista, 12.IX.1932, fl., P. Capucho 432 (F-scanned image); Conceição do Araguaia, range of low hills ca. $20 \mathrm{~km}$ west of Redenção, near Corrego São João and Troncamento Santa Teresa, 11.II.1980, fr., T. Plowman et al. 8703 (Fscanned image, IAN, INPA, MG); Concórdia do Pará, Cristo Rei, 15.I.2002, fr., M.R. Cordeiro MC-19-10 (100.471) (IAN); Marabá, Rio Itacaiunas, afl. do Rio Tocantins, Serra Buritirama (B 5), região com minério de manganês, árvore 526-13, VIII.1970, st., J.M. Pires \& R.P. Belem 12736 (IAN); Melgaço, Estação Científica Ferreina Penna, trilha central, 24. VI.2004, fl., J. Oliveira et al. 868 (MG); Oriximiná, Lago Salgado (Baixo Trombetas), 22.IV.1917, st., A. Ducke s.n. (RB 22444); Pau D'Arco, Santana do Araguaia, 21.VIII.1998, st., J. Grogan 605 (IAN); Porto de Moz, Rio Perí, afl. do Xingu, região onde foi feito um levantamento estatístico florestal pelo IAN, SPVEA e FAO, 29.XI.1955, fl., R.L. Fróes 32430 (IAN); São João do Araguaia, Parque da Serra dos Martírios (Andorinhas), 18.IX.2002, st., M.R. Cordeiro MC-29-18 (100.587) (IAN); Tucuruí, estrada para Repartimento, km 25, 4.VI.1980, fr., M.G. Silva \& C. Rosário 5364 (INPA, MG); Vitória do Xingu, Usina Hidrelétrica Belo Monte, 23.X.2012, fl., C. Faveri PSACF 756 (MG).
Additional material: BRAZIL, AMAPÁ: Mazagão, área do Felipe, 3.VII.1984, f1., N.T. Silva 5381 (MG); AMAZONAS: Manaus, Reserva Florestal Ducke, Manaus-Itacoatiara, km 26, 29.V.1995, fl., P.A.C.L. Assunção \& E.C. Pereira 201 (MG); RORAIMA: Alto Alegre, Estação Ecológica de Maracá, Grade do PPBio, trilha N01, segmento 1000-1100, 18.V.2012, fr., R.O. Perdiz et al. 1361 (INPA).

Phytoecological region and phenology: Dense Ombrophilous Forest (terra firme forest). Flowers and fruits collected from June to February.

Common names: araracanga, araracanga-preta, jararacanga, muirapyranga.

According to Woodson (1951), Aspidosperma eteanum is a species endemic to Brazil, and occurs in the states of Amapá, Amazonas, Maranhão, Pará and Roraima (Missouri Botanical Garden 2015). In the state of Pará, this species was found in Baixo Amazonas, Marajó, Nordeste Paraense, Sudeste Paraense and Sudoeste Paraense Mesoregions (IBGE 1990). MarcondesFerreira (1988) reduced Aspidosperma eteanum to be synonymous with $A$. desmanthum (section Nobilia), but we decided to keep it as an accepted species due to observed differences between the specimens of the two species. Aspidosperma eteanum resembles $A$. desmanthum, especially leaf traits, but differs from A. desmanthum by the prominulous secondary veins on the abaxial surface, tubular corolla, length of corolla lobes (1-1.5 $\mathrm{mm}$ long) and ovate seeds.

9. Aspidosperma excelsum Benth., J. Bot. (Hooker) 3: 245. 1841. (Figure 4a-e).

Trees 18-48 m high; trunk tortuous. Branches cylindrical, not suberous, sparsely lenticellate, pubescent to glabrous, without cataphylls; latex whitish. Leaves alternate, arranged along the branches; petioles 1.2-1.9 cm long, tomentose; blade 7-8.9 x 2.5$3.4 \mathrm{~cm}$, coriaceous, flat, elliptic or obovate, apex acute, obtuse or retuse, base cuneate or oblique, margin revolute, discolorous, venation brochidodromous, without marginal vein, adaxial surface lustrous, dark brown, glabrous, primary vein flat, secondary veins impressed, tertiary veins inconspicuous, abaxial surface dull, light brown, tomentose along the primary vein, primary vein prominent, secondary veins impressed, 22-24 pairs, tertiary veins inconspicuous. Inflorescences $5.7-6.5 \mathrm{~cm}$ long, supra-axillary or terminal, corymbiform dichasia, tomentose. Flower buds with corolla lobes not twisted. Flowers $6.2-7.5 \mathrm{~mm}$ long; pedicellate, pedicel 1.5-2 x $1 \mathrm{~mm}$, tomentose. Calyx 2.5-3 x $2.5 \mathrm{~mm}$, tomentose externally, pubescent at apex internally; lobes 5, 1.5-3 x 1-1.5 mm, ovate, apex acute or obtuse. Corolla 5.5-6.2 x $1.5 \mathrm{~mm}$, tubular, white, tomentose externally, tomentose below the anthers internally; lobes $1-1.5 \times 0.3 \mathrm{~mm}$, patent, ovate, apex acute. Stamens 3-4 mm long; filaments 2.5-3.2 $\mathrm{mm}$ long, tomentose; anthers 0.5-0.8 mm long, ovate, apex acute. Carpels 2.9-3.9 mm long; ovary 0.5-1 x $0.5 \mathrm{~mm}$, ovoid, glabrous; style 2-2.5 mm long; style-head $0.4 \mathrm{~mm}$ long, main body oblong, with 2 oblong apical appendages. Follicles 4.5-6 x 4-5 cm, dolabriform, spinescent, stipitate, mucronate, lenticels inconspicuous, brown, pubescent. Seeds $4-5 \mathrm{~cm}$ diam., orbicular; seminal nucleus central, without radial lines, nucleus $1.2 \mathrm{~cm}$ diam.

Material examined: BRAZIL, PARÁ: Almeirim, região do Rio Jarí, Planalto de Monte Alegre, 5.IX.1968, fl., E. Oliveira 4841 (IAN, NY-scanned image); Altamira, Xingu River, Assurini indians, 13.VI.1986, st., W. Balée 2491 (NY-scanned image); Belém, Jardim Botânico da Amazônia Bosque Rodrigues Alves, canteiro 75, árvore $\mathrm{n}^{\circ}$ 2270, 24.IX.2014, fr., A.S. de S. Pereira 97 (MG); Breves, local onde foi feito um inventário 
florestal, X-XI.1957, fr., J.M. Pires \& N.T. Silva 6645 (IAN); Concórdia do Pará, Sítio São José, 13.II.2002, st., M.R. Cordeiro MC-24-02 (100.510) (IAN); Gurupá, 19.VIII.1918, st., A. Ducke s.n. (MG 17224); Ipixuna, 7.VII.1966, st., A.P. Duarte 9804 (RB); Itaituba, 27.VI.1972, st., J.B. Rodrigues 53 (R); Jacareacanga, Rio Tapajós, estrada das cachoeiras, lugar Periquito, 7. XII.1915, st., A. Ducke s.n. (MG 15860); Mãe do Rio, Vila Mãe do Rio, 26.III.1998, st., N.A. Rosa 5744 (MG); Marituba, mata da Cia. Pirelli, Fazenda Uribóca, 12.IX.1958, fl., J.M. Pires 7141 (IAN); Moju, campo experimental da Embrapa Amazônia Oriental no $\mathrm{km} 30$ da rodovia PA-150, margem do ramal atrás do $3^{\circ}$ pátio da exploração, 13.II.1998, st., G.C. Ferreira \& J.C. Freitas 422 (IAN); Monte Alegre, Macau airstrip, 1 1/2 hrs. upstream from Lageira airstrip, on Rio Maicurú, 28.VII.1981, st., J.J. Strudwick et al. 3632 (MG); Oriximiná, 3.III.1915, fr., A. Ducke s.n. (MG 15703); Pau D’Arco, Marajoara, s.d., st., J. Grogan 22 (IAN); Santa Bárbara, entrada do Genipaúba, 9. V.2013, fr., R.C.S. Trindade \& M.R.C. Ferreira 1 (MG, UEC); Santana do Araguaia, $100 \mathrm{~km}$ south of Redenção on road (PA150) to Barreiras dos Campos, Fazenda Inajaporã between Rio Inajazinho and Rio Inajá, 19.II.1980, fr., T. Plowman et al. 8943 (INPA, MG); Santarém, Reserva Curuá-Una, Parque Fenológico, árvore $\mathrm{n}^{\circ}$ 173, 14.VI.1999, st., M.R. Cordeiro \& N.J. Moraes 3900 (IAN); Tucuruí, Transgoiânia, área nuclear $\mathrm{n}^{\circ} 4$, III.1981, st., P. Lisboa et al. 2451 (MG).

Additional material: BRAZIL, AMAZONAS: Manaus, Cachoeira Baixa do Tarumã, margem inundada ao pé da cachoeira, 9.IX.1940, fl., A. Ducke 624 (MG); Manaus, 24. XII.1942, fr., A. Ducke 1163 (NY-scanned image); Manaus, estrada da Reserva Florestal Ducke, 7.III.1958, fr., Pessoal do C.P.F. s.n. (RB 125179).

Phytoecological region and phenology: Dense Ombrophilous Forest (terra firme forest). Flowers and fruits collected from September to May.

Common names: carapanaúba, carapanaúba-amarela, carapanaúba-do-baixio, carapanaúba-da-terra-firme, kina, pepembyra.

Aspidosperma excelsum is a widely distributed species, occurring in Bolivia, Brazil, Colombia, Costa Rica, French Guiana, Guyana, Panama, Peru, Suriname and Venezuela (Missouri Botanical Garden 2015). In the state of Pará, this species was found in all Mesoregions (IBGE 1990). According to Marcondes-Ferreira \& Kinoshita (1996), Aspidosperma excelsum belongs to section Excelsa. It shares morphological features with Aspidosperma oblongum, especially flower structures, but can be differentiated from $A$. oblongum by the glabrous ovary, spinescent follicles and leaves without a marginal vein.

10. Aspidosperma inundatum Ducke, Arch. Jard. Bot. Rio de Janeiro 3: 245. 1922. (Figure 4f-j).

Trees 10-18 m high; trunk straight. Branches cylindrical, not suberous, sparsely lenticellate, pubescent to glabrescent, without cataphylls; latex whitish. Leaves alternate, arranged along the branches; petioles 1-1.5 cm long, pubescent; blade 7.2-8.5 x 3-5 $\mathrm{cm}$, chartaceous to subcoriaceous, flat, oblong or elliptic, apex acuminate or acute, base cuneate or oblique, margin revolute, discolorous, venation brochidodromous, with marginal vein $1.2 \mathrm{~mm}$ from the margin, adaxial surface lustrous, dark brown, glabrous, primary vein flat, secondary veins impressed, tertiary veins inconspicuous, abaxial surface dull, light brown, pubescent to glabrescent, primary vein prominent, secondary veins prominulous, 23-25 pairs, tertiary veins inconspicuous. Inflorescences $6.1-7 \mathrm{~cm}$ long, axillary or supra-axillary, corymbiform dichasia, tomentose.
Flower buds with corolla lobes not twisted. Flowers 17.5-18.5 mm long; pedicellate, pedicel 2-3 x $1 \mathrm{~mm}$, tomentose. Calyx 3-3.5 x 2.1-2.5 mm, tomentose externally, pubescent at apex internally; lobes 5, 2-2.1 × $2 \mathrm{~mm}$, ovate, apex acute. Corolla 15-15.7 × $2 \mathrm{~mm}$, salverform, white, tomentose externally, pubescent along the corolla internally; lobes 9.7-10 x $2 \mathrm{~mm}$, erect or deflexed, oblong or lanceolate, apex acute or obtuse. Stamens 5.5-6 mm long; filaments 3.5-4 mm long, tomentose; anthers $2 \mathrm{~mm}$ long, lanceolate, apex apiculate or acute. Carpels 3.5-3.8 mm long; ovary 2-2.3 x 1-1.5 mm, ovoid, tomentose; style $1 \mathrm{~mm}$ long; stylehead $0.5 \mathrm{~mm}$ long, main body oblong, with 2 filiform apical appendages. Follicles 8-8.5 x $6 \mathrm{~cm}$, dolabriform, smooth, sessile, mucronate, lenticels conspicuous, black, glabrous. Seeds 7.1-7.5 $\mathrm{x}$ 6.1-5.7 cm, oblong; seminal nucleus central, without radial lines, nucleus $3.6 \mathrm{~cm}$ diam.

Material examined: BRAZIL, PARÁ: Breves, Antônio Lemos, Rio Amazonas, Igarapé Pixuna, beira do Rio Tajapurú, 19.VII.1948, fl., G.A. Black 48-2946 (IAC, IAN); Gurupá, silva ab Amazonum fluvio inundata, 13.VIII.1918, fl., A. Ducke s.n. (NY 297971-scanned image, RB 15814); Oriximiná, Lago Salgado, Rio Trombetas, 9.IX.1927, fl., A. Ducke s.n. (RB 21808).

Additional material: BRAZIL, AMAPÁ: Macapá, Rio Carapanã Branca, 6.I.1977, fr., E. Oliveira 6530 (MG).

Phytoecological region and phenology: Dense Ombrophilous Forest (várzea forest). Flowers and fruits collected from July to January.

Common names: araracanga, jacamiramane, maparaná.

According to Koch et al. (2015), Aspidosperma inundatum is a species endemic to Brazil, and occurs in the states of Amapá, Amazonas, Mato Grosso and Pará. In the state of Pará, this species was found in Baixo Amazonas and Marajó Mesoregions (IBGE 1990). In Marcondes-Ferreira \& Kinoshita (1996), Aspidosperma inundatum is placed in the monospecific section Inundata. Aspidosperma inundatum was one of the most easily recognized species, and can be differentiated by the larger flowers (17.5-18.5 mm long) and the sessile black follicles with conspicuous lenticels.

11. Aspidosperma macrocarpon Mart., Flora 7(1): 136. 1824. (Figure 4k-o).

Trees 3-35 m high; trunk straight. Branches cylindrical, not suberous, sparsely lenticellate, velutinous to tomentose, without cataphylls; latex whitish. Leaves alternate, arranged along the branches; petioles $1-4.5 \mathrm{~cm}$ long, velutinous to tomentose; blade 9-20.1 x 7-15 cm, chartaceous, flat, oblong or ovate, apex acute or obtuse, base cuneate or oblique, margin straight or revolute, discolorous, venation eucamptodromous, adaxial surface dull or lustrous, dark brown, velutinous, tomentose or pubescent, primary vein prominulous or prominent, secondary veins prominulous, tertiary veins conspicuous, abaxial surface dull, light brown, velutinous to tomentose, primary vein prominent, secondary veins prominent, 10-13 pairs, tertiary veins conspicuous. Inflorescences 5-7 cm long, axillary or terminal, corymbiform dichasia, velutinous to tomentose. Flower buds with corolla lobes not twisted. Flowers 15-20 mm long; pedicellate, pedicel 2$2.5 \times 1 \mathrm{~mm}$, tomentose. Calyx 2.5-3 x 3-3.5 mm, tomentose externally, pubescent at apex internally; lobes $5,1.5 \times 1 \mathrm{~mm}$, ovate, apex acute. Corolla 15-16 x $2 \mathrm{~mm}$, salverform, white, tomentose externally, pubescent to glabrescent along the corolla internally; lobes 10-12 × $4 \mathrm{~mm}$, erect or deflexed, oblong or obovate, apex obtuse. Stamens 5-6 mm long; filaments 3.5-4.5 $\mathrm{mm}$ long, pubescent to glabrescent; anthers $1.5 \mathrm{~mm}$ long, ovate, 


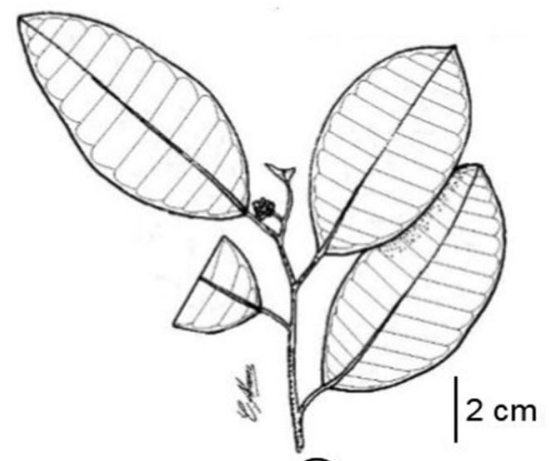

(a)
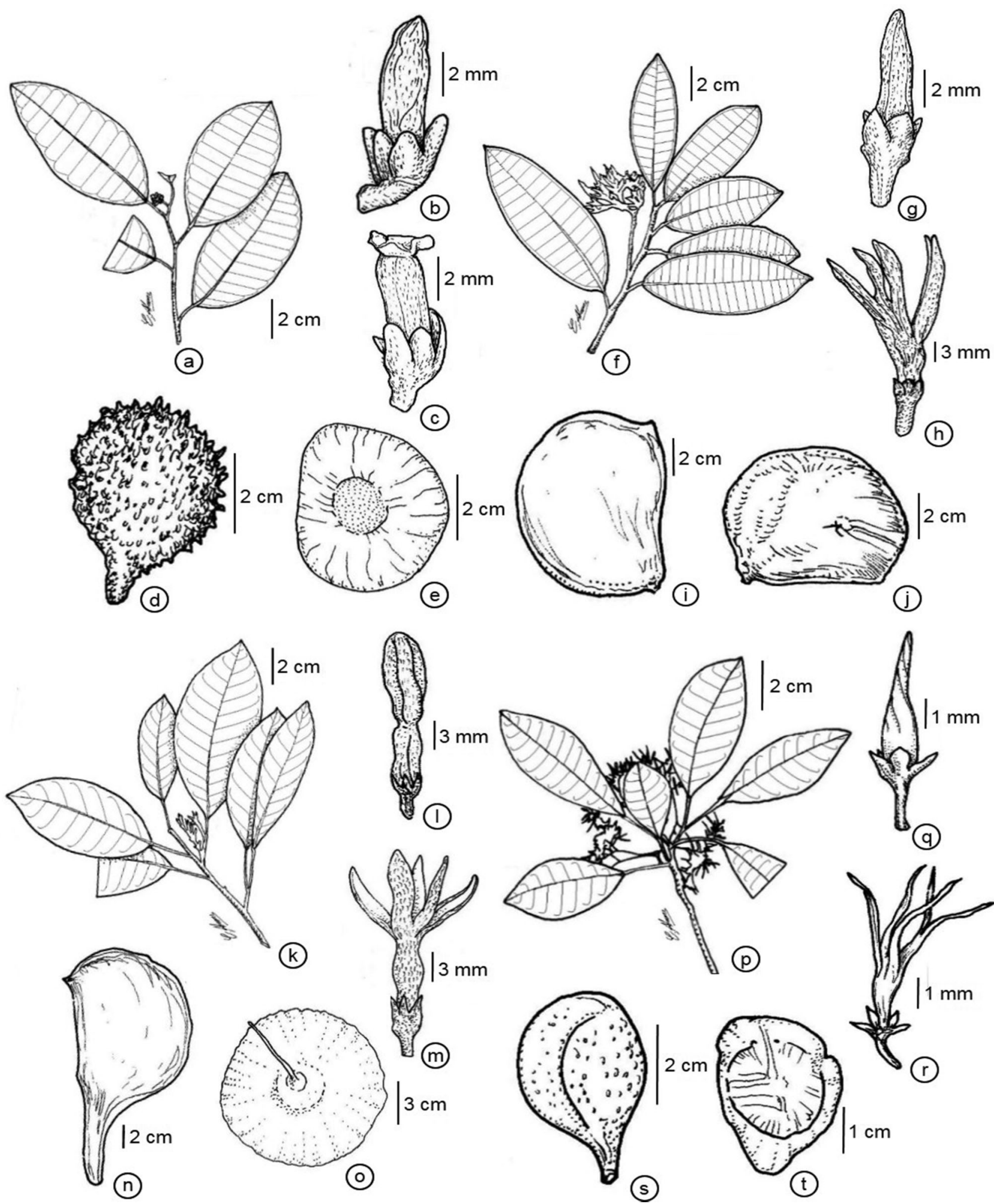

Figure 4. a-e. Aspidosperma excelsum. f-j. A. inundatum. k-o. A. macrocarpon. p-t. A. multiflorum: a, f, k, p. flowering branch; b, g, l, q. flower bud; c, h, m, r. flower; d, i, n, s. follicle; e, j, o, t. seed. a-e. A. Ducke 624; A. Ducke 1163; Pessoal do C.P.F. s.n. (RB 125179). f-j. A. Ducke s.n. (NY 297971, RB 15814); G.A. Black 48-2946. k-o. C.A. Cid et al. 1378; C.R. Sperling et al. 6248; U.N. Maciel \& M.R. Cordeiro 120. p-t. A.M. Miranda et al. 5310; G.A. Black 47-1740.

Figura 4. a-e. Aspidosperma excelsum. f-j. A. inundatum. k-o. A. macrocarpon. p-t. A. multiflorum: a, f, k, p. ramo florido; b, g, l, q. botão floral; c, h, m, r. flor; d, i, n, s. folículo; e, j, o, t. semente. a-e. A. Ducke 624; A. Ducke 1163; Pessoal do C.P.F. s.n. (RB 125179). f-j. A. Ducke s.n. (NY 297971, RB 15814); G.A. Black 48-2946. k-o. C.A. Cid et al. 1378; C.R. Sperling et al. 6248; U.N. Maciel \& M.R. Cordeiro 120. p-t. A.M. Miranda et al. 5310; G.A. Black 47-1740. 
apex apiculate. Carpels $3.7 \mathrm{~mm}$ long; ovary 1 x $1 \mathrm{~mm}$, globoid, glabrous; style $2 \mathrm{~mm}$ long; style-head $0.7 \mathrm{~mm}$ long, main body oblong, with 2 oblong apical appendages. Follicles 8.2-12 x 7-10.4 cm, dolabriform, smooth, stipitate, mucronate, lenticels inconspicuous, brown, velutinous to pubescent. Seeds $8.2-10 \mathrm{~cm}$ diam., orbicular; seminal nucleus central, without radial lines, nucleus $4 \mathrm{~cm}$ diam.

Material examined: BRAZIL, PARÁ: Almeirim, Monte Dourado, área do Ideal, 14.X.1980, fl., N.T. Silva 5448 (INPA, MG); Altamira, região Garotire, 8.VIII.1962, fl., N.T. Silva 786 (IAN); Belterra, beira do Rio Tapajós, Pindobal, 27. X.1947, fr., G.A. Black 47-1827 (IAC, IAN); Belterra, margem da estrada Pindobal-Porto Novo, 9.XII.1978, fl., R. Vilhena et al. 182 (MG, NY-scanned image); Faro, Terra Santa, 4. VII.1975, st., S. Assunpção \& D. Coelho 35 (INPA); Monte Alegre, Rio Maicurú, mata entre Caá-ussú e localidade de Balança, 16.IX.1953, fl., R.L. Fróes 30242 (IAN); Óbidos, 20. X.1919, fl., A. Ducke s.n. (RB 11402); Oriximiná, Rio Trombetas, margem da estrada, mineração Santa Patricia, 8. VII.1980, fr., C.A. Cid et al. 1378 (INPA, MG); Parauapebas, Serra dos Carajás, $10 \mathrm{~km}$ east of AMZA camp N-1 and 5-10 $\mathrm{km}$ along the entrance road to Azul, an abandoned manganese exploration camp, 19.VI.1982, fl., C.R. Sperling et al. 6248 (MG); Santarém, beira da estrada que liga Belterra a Porto Novo, 4.XII.1978, fr., M.G.A. Lobo et al. 108 (MG, NYscanned image); Santarém, estrada de Belterra, 6.X.1962, fl., A. P. Duarte 7016 (RB); Santarém, Porto Novo-Aramanai, 2. XII.1978, fr., U.N. Maciel \& M.R. Cordeiro 120 (MG); São Geraldo do Araguaia, morro 3, 15.VI.1995, fr., M.N. Bastos \& M.R. Cordeiro 2015 (IAN, MG).

Additional material: BRAZIL, MATO GROSSO: Cuiabá, X.1914, st., J.G. Kuhlmann 1227 (R); Entroncamento das rodovias, Cuiabá-Santarém e Porto Velho, arredores do aeroporto, 4.II.1979, fr., M.G. Silva \& A. Pinheiro 4432 (MG); Sacaré, campo base do RADAMBRAZIL, folha SD 21YC, rodovia BR-416 entre Pontes de Lacerda e Mato Grosso (ex Vila Bela), 2.VIII.1978, fr., J.M. Pires \& M.R. Santos 16347 (MG); RONDÔNIA: Basin of Rio Madeira, Serra dos Murales, $14 \mathrm{~km} \mathrm{NNW}$ of junction of Rios Madeira and Abunã, 14.VII.1968, fl., G.T. Prance et al. 6030 (MG).

Phytoecological region and phenology: Dense Ombrophilous Forest (terra firme forest) and Savannah. Flowers and fruits collected from June to December.

Common names: marajussara, muirajuçara, muirajussara, peroba, peroba-branca, peroba-mico.

The information about the protologue of Aspidosperma macrocarpon was checked in its original publication. The guide "Taxonomic Literature" (Stafleu \& Cowan 1981), also states that Aspidosperma macrocarpon was first described in "Flora" by Martius (1824a) and not in "Nova Genera et Species Plantarum" by the same author (Martius 1824b). In the databases "Tropicos" (Missouri Botanical Garden 2015) and "World Checklist of Selected Plant Families" (WCSP 2015), this information is conflicting. Aspidosperma macrocarpon occurs in Bolivia, Brazil, Peru and Venezuela (Missouri Botanical Garden 2015). In the state of Pará, this species was found in Baixo Amazonas, Sudeste Paraense and Sudoeste Paraense Mesoregions (IBGE 1990). According to MarcondesFerreira \& Kinoshita (1996), Aspidosperma macrocarpon belongs to section Aspidosperma. This species can be easily differentiated from the other species in section Aspidosperma occurring in Pará by the larger follicles $(8.2-12 \times 7-10.4 \mathrm{~cm})$ with inconspicuous lenticels and branches without cataphylls covering the buds.

12. Aspidosperma multiflorum A.DC. in A.P.de Candolle, Prodr. 8: 397. 1844. (Figure 4p-t).

Trees 2-22 m high; trunk straight. Branches cylindrical, not suberous, densely lenticellate, pubescent to glabrescent, with cataphylls; latex whitish. Leaves alternate, congested at apex of branches; petioles $2-3 \mathrm{~cm}$ long, pubescent to glabrous; blade 5.5-6.3 x 3.5-4.5 cm, membranaceous, flat, elliptic, apex acuminate or acute, base attenuate or oblique, margin straight, concolorous, venation eucamptodromous, adaxial surface dull, light brown, pubescent to glabrous along the primary vein, primary vein flat, secondary veins prominulous, tertiary veins inconspicuous, abaxial surface dull, light brown, pubescent to glabrous along the primary vein, primary vein flat or prominent, secondary veins prominulous, $14-18$ pairs, tertiary veins conspicuous. Inflorescences $4-5.3 \mathrm{~cm}$ long, terminal, corymbiform dichasia, tomentose. Flower buds with corolla lobes twisted. Flowers 6-6.5 mm long; pedicellate, pedicel 1-1.3 x $0.2 \mathrm{~mm}$, glabrous. Calyx 0.5-1 x $1 \mathrm{~mm}$, glabrous externally, pubescent at apex internally; lobes 5, 0.5-0.8 x 0.3-0.5 mm, ovate, apex acute. Corolla $5 \times 0.5 \mathrm{~mm}$, salverform, orange, glabrous externally, pubescent along the corolla internally; lobes 3.5-4 x $0.2 \mathrm{~mm}$, erect or deflexed, filiform, apex acute. Stamens 1.8-2 mm long; filaments 1.3-1.5 mm long, pubescent; anthers $0.5 \mathrm{~mm}$ long, ovate, apex acute. Carpels $1.6 \mathrm{~mm}$ long; ovary $0.5 \times 0.5 \mathrm{~mm}$, globoid, glabrous; style $0.8 \mathrm{~mm}$ long; stylehead $0.3 \mathrm{~mm}$ long, main body oblong, with 2 ovate apical appendages. Follicles 3-4 x 2-3 cm, pyriform or suborbicular, smooth, stipitate, mucronate or not, lenticels conspicuous, brown, glabrous. Seeds 3-3.5 x 2.2-2.5 cm, ovate; seminal nucleus central, without radial lines, nucleus $1.5-2 \mathrm{~cm}$ diam.

Material examined: BRAZIL, PARÁ: Belterra, beira do Rio Tapajós, 24.X.1947, fr., G.A. Black 47-1740 (IAC, IAN); Belterra, estrada Porto Novo-Pindobal, 7.XII.1978, f1., M.G. A. Lobo et al. 204 (IAN, MG); Canaã dos Carajás, 27. XII.2000-6.I.2001, fr., L.C.B. Lobato et al. 2618 (MG); Faro, Campina do Jupiry (Lago de Faro), 25.I.1927, st., A. Ducke s. n. (RB 148804); Monte Alegre, 11.III.1923, st., A. Ducke s.n. (RB 22436); Santarém, região Rio do Tapajós, IX.1957, fl., R. L. Fróes 33564 (IAN); Santarém, região do Planalto de Santarém, onde foi feito um levantamento estatístico florestal pelo IAN, SPVEA e FAO, 28.X.1954, fl., R.L. Fróes 31179 (IAN, RB); Santarém, Taperinha, Paraná do Ituquí, região do Planalto de Santarém, 3.XII.1954, fl., R.L. Fróes 31205 (IAN, R); Santarém, Vila de Alter do Chão, praia arenosa do Rio Tapajós, depois do Lago Jacundá, em direção ao Lago do Pindobal, 4.I.1992, fr., L.V. Ferreira \& A.L.K.M Albernaz 73 (INPA); Tucuruí, approx. 30-35 km from Tucuruí, $25 \mathrm{~km}$ on old BR-422, then 5-10 km E on old railroad bed., 31.X.1981, fl., D.C. Daly et al. 1114 (INPA, MG).

Additional material: BRAZIL, MARANHÃO: Approx. 40 km E of Barra do Corda toward Presidente Dutra, 10.X.1980, fl., D.C. Daly et al. D568 (MG); MATO GROSSO: Nobres, BR-163 Cuiabá-Sinop a $145 \mathrm{~km}$ ao S de Sinop, próximo a Lucas do Rio Verde, 17.IX.1985, fl., C.A.C. Ferreira et al. 6074 (MG); PIAUÍ: Guadalupe, margem da barragem de Boa Esperança, 22.XI.2005, fl., A.M. Miranda et al. 5310 (RB); RONDÔNIA: Vicinity of

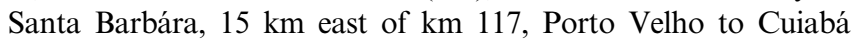
highway, 12.VIII.1968, fl., G.T. Prance \& J.F. Ramos 6882 (MG).

Phytoecological region and phenology: Savannah. Flowers and fruits collected from August to April. 
Common names: muirajussára-hy.

According to Koch et al. (2015), Aspidosperma multiflorum is a species endemic to Brazil, and occurs in more than half of the states. In the state of Pará, this species was found in Baixo Amazonas and Sudeste Paraense Mesoregions (IBGE 1990). In Marcondes-Ferreira \& Kinoshita (1996), Aspidosperma multiflorum is placed in section Aspidosperma. This species resembles Aspidosperma pyrifolium especially fruit morphology, but differs from $A$. pyrifolium by the membranaceous leaves, smaller flowers (6-6.5 mm long), filiform corolla lobes and externally glabrous calyx.

13. Aspidosperma oblongum A.DC. in A.P.de Candolle, Prodr. 8: 399. 1844. (Figure 5a-e).

Trees 13-42 m high; trunk tortuous. Branches cylindrical, not suberous, densely lenticellate, pubescent to glabrescent, without cataphylls; latex whitish. Leaves alternate, arranged along the branches; petioles $0.7-2.1 \mathrm{~cm}$ long, glabrous; blade 5-8.1 x 2.3-3 cm, chartaceous, flat, oblong, apex acuminate or acute, base revolute, margin straight or revolute, discolorous, venation brochidodromous, with marginal vein $1 \mathrm{~mm}$ from the margin, adaxial surface lustrous, black, glabrous, primary vein flat, secondary veins prominent, tertiary veins inconspicuous, abaxial surface dull, light brown, pubescent, primary vein prominent, secondary veins prominent, 24-26 pairs, tertiary veins inconspicuous. Inflorescences $6.1-14 \mathrm{~cm}$ long, terminal, corymbiform dichasia, tomentose. Flower buds with corolla lobes not twisted. Flowers 7-8.1 mm long; pedicellate, pedicel 2.7-3.5 x 0.5-0.7 mm, tomentose. Calyx 1.8-2 x 1.5-2 mm, tomentose externally, pubescent at apex internally; lobes 5, 0.8$1 \times$ 0.5-0.7 mm, ovate, apex acute or obtuse. Corolla 4.5-4.7 x $1.5 \mathrm{~mm}$, tubular, white, tomentose externally, pubescent to glabrescent along the corolla internally; lobes $1-1.5 \times 0.5 \mathrm{~mm}$, patent, ovate, apex acute. Stamens $2.5-3 \mathrm{~mm}$ long; filaments 2-2.2 mm long, pubescent to glabrescent; anthers $0.5-0.8 \mathrm{~mm}$ long, ovate, apex apiculate. Carpels 1.4-2.1 mm long; ovary 0.5-0.7 x $0.7 \mathrm{~mm}$, ovoid, tomentose; style $0.5-1 \mathrm{~mm}$ long; stylehead $0.4 \mathrm{~mm}$ long, main body oblong or globose, with 2 oblong apical appendages. Follicles 4.4-6 x 3.2-4 cm, suborbicular, verrucose, stipitate, not mucronate, lenticels inconspicuous, brown, pubescent. Seeds $4 \mathrm{~cm}$ diam., orbicular; seminal nucleus central, without radial lines, nucleus $2 \mathrm{~cm}$ diam.

Material examined: BRAZIL, PARÁ: Almeirim, região do Rio Jarí, estrada entre Planalto B e Braço, 12.IV.1969, fr., N.T. Silva 1855 (IAN); Almeirim, região do Rio Jarí, Monte Dourado, Planalto B, 21.X.1968, fl., N.T. Silva 1285 (IAN); Belterra, 25.VII.1947, st., G. Black 47-1075 F25 (IAC, IAN, UEC); Itaituba-Santarém, árvore $n^{\circ}$ 29-3-13, XII.1972, fr., J. M. Pires 13820 (IAN); Melgaço, Floresta Nacional de Caxiuanã, local onde será construída a Estação Científica "Ferreira Penna", 2-15.II.1991, st., A.S.L. da Silva et al. 2313 (MG); Melgaço, Floresta Nacional de Caxiuanã, trilha que dá acesso a área do projeto ESECAFLOR, 2.IX.2014, f1., A.S. de S. Pereira et al. 96 (MG); Santarém, Lago Cuçarí, região do Planalto de Santarém, onde foi feito o levantamento estatístico florestal pelo IAN, SPVEA e FAO, 13.V.1955, st., R.L. Fróes 31817 (IAN); Tucuruí, A.N.5, próx. Rio Macoari, margem direita do Rio Tocantins, 10.IV.1981-29.V.1981, st., U.N. Maciel et al. 649 (MG); Vitória do Xingu, Sítio Pimental, 26. IV.2013, fl., F.A. Raul PSACF 1077 (MG).

Additional material: BRAZIL, AMAPÁ: Rio Araguari, camp 13, 5.X.1961, fl., J.M. Pires et al. 51510 (RB); AMAZONAS: Manaus, Reserva Florestal Ducke, Q III, árv.
352, 30.VII.1964, fr., W. Rodrigues \& Osmarino 5980 (RB); Manaus-Itacoatiara km 31, 17.IX.1962, fl., A.P. Duarte \& Gilbert 6924 (RB). FRENCH GUIANA: s.d., fr., R. Benoist 963 (P-scanned image); Caiena, s.d., fl., Martin s.n. (P 4205125scanned image).

Phytoecological region and phenology: Dense Ombrophilous Forest (terra firme forest). Flowers and fruits collected from September to April.

Common names: carapanaúba, carapanaúba-branca, carapanaúba-legítima.

Aspidosperma oblongum occurs in Brazil, French Guiana, Guyana, Suriname and Venezuela (Missouri Botanical Garden 2015). In the state of Pará, this species was found in Baixo Amazonas, Marajó, Sudeste Paraense and Sudoeste Paraense Mesoregions (IBGE 1990). Marcondes-Ferreira (1988) reduced Aspidosperma oblongum to be synonymous with $A$. discolor (section Excelsa), but based on observed differences between the specimens of the two species, we decided to recognize A. oblongum as an accepted species. Aspidosperma oblongum shares morphological features with both $A$. discolor and A. excelsum, mainly flower structures, but differs from the former by the oblong leaf blades with revolute base, ovate anthers and verrucose follicles, and from the latter by leaves with a marginal vein, tomentose ovary and verrucose follicles.

14. Aspidosperma pyrifolium Mart., Flora 7(1): 136. 1824. (Figure 5f-j).

Trees 3-12 m high; trunk straight. Branches cylindrical, not suberous, densely lenticellate, glabrescent to glabrous, with cataphylls; latex whitish. Leaves alternate, congested at apex of branches; petioles $0.7-2 \mathrm{~cm}$ long, tomentose, pubescent or glabrescent; blade 6-9.7 x 2.5-4 cm, chartaceous, flat, oblong, elliptic or ovate, apex acuminate, acute or obtuse, base attenuate or oblique, margin revolute, concolorous, venation eucamptodromous, adaxial surface dull or lustrous, dark brown, pubescent to glabrescent, primary vein flat, secondary veins prominent, tertiary veins inconspicuous, abaxial surface dull, dark brown, velutinous to glabrescent, primary vein prominent, secondary veins prominent, 12-17 pairs, tertiary veins conspicuous. Inflorescences $3.4-6.5 \mathrm{~cm}$ long, terminal, corymbiform dichasia, tomentose. Flower buds with corolla lobes twisted. Flowers 14-23 mm long; pedicellate, pedicel 1-5 x 0.5-1 mm, pubescent. Calyx 0.5-1 x $1.8 \mathrm{~mm}$, tomentose externally, glabrous internally; lobes $5,2-3 \times 0.5-0.7 \mathrm{~mm}$, lanceolate, apex acuminate. Corolla $12-15 \times 1.2 \mathrm{~mm}$, salverform, white, pubescent externally, pubescent at base internally; lobes 10-13 x $3 \mathrm{~mm}$, deflexed, oblong, apex acute. Stamens 2-2.5 mm long; filaments 1-1.5 mm long, pubescent; anthers $1 \mathrm{~mm}$ long, ovate, apex acute. Carpels $2 \mathrm{~mm}$ long; ovary $0.7 \mathrm{x}$ $0.5 \mathrm{~mm}$, globoid, glabrous; style $1 \mathrm{~mm}$ long; style-head $0.3 \mathrm{~mm}$ long, main body globose, with 2 oblong apical appendages. Follicles 4.3-5 x 2-2.5 cm, pyriform or suborbicular, smooth, stipitate, mucronate or not, lenticels conspicuous, brown, glabrous. Seeds 3.5-6 x 2.2-4.7 cm, ovate; seminal nucleus central, without radial lines, nucleus $1.5-2.2 \mathrm{~cm}$ diam.

Material examined: BRAZIL, PARÁ: Belterra, Pindobal, 23. XII.1956, fr., J.M. Pires et al. 6512 (IAN); Faro, Campina do Jupiry, 17.VI.1926, st., A. Ducke s.n. (RB 21801); Monte Alegre, Parque Estadual, base da Serra do Ererê, 18.IV.2006, fr., A.E.S. Rocha 470 (MG); Santarém, centro de treinamento da FAO, 5. X.1962, fr., A.P. Duarte 7015 (INPA, RB); Santarém, Lago Cuçarí, região do Planalto de Santarém, onde foi feito o levantamento estatístico florestal pelo IAN, SPVEA e FAO, 
V.1955, fr., R.L. Fróes 31825 (IAN, RB); Santarém, Rio CuruáUna, região do Planalto de Santarém, onde foi feito o levantamento estatístico florestal pelo IAN, SPVEA e FAO, 14. XI.1954, fr., R.L. Fróes 31394 (IAN).

Additional material: BRAZIL, BAHIA: Canudos, estrada em direção a Jeremoabo, ca. 17,8 km de Canudos, 953'18"S, 38 51'54"W, 28.VI.2002, fr., L.P. Queiroz et al. 7261 (RB); Jeremoabo, ca. $23 \mathrm{~km}$ E de Canudos na estrada para Jeremoabo (BR-235), 26.VIII.1996, fr., L.P. Queiroz \& N.S. Nascimento 4647 (INPA); Rui Barbosa, trilha para o Pátio das Orquídeas, $12^{\circ}$ 18'7”S, 40²9'16"W, 12.XI.2004, fl., A. Rapini \& L.P. Queiroz 1156 (INPA); MATO GROSSO: Rio Juruena, beira do rio, 15. VII.1977, fr., M.G. Silva \& J. Maria 3357 (MG); XavantinaCachimbo road, cerradão c. $0.5 \mathrm{~km} \mathrm{~W}$ of $\mathrm{km} \mathrm{264}$, expedition base camp, 20.III.1968, fr., D. Philcox \& A. Ferreira 4594 (RB); RONDÔNIA: Vilhena, estrada que vai para Aripuanã, local Fazenda Flor da Serra, a $15 \mathrm{~km}$ de Vilhena, 22.V.1984, fr., C.S. Rosário et al. 475 (MG); Médici, Picadão que confina a $7^{\mathrm{a}}$ e a $8^{\mathrm{a}}$ linha, margem esquerda da BR-429, 24.III.1986, fr., N.A. Rosa et al. 4985 (MG).

Phytoecological region and phenology: Savannah. Flowers and fruits collectted from March to December.

Common names: mirajussara, perobinha.

The information about the protologue of Aspidosperma pyrifolium was checked in its original publication. The guide "Taxonomic Literature" (Stafleu \& Cowan 1981), also states that Aspidosperma pyrifolium was first described in "Flora" by Martius (1824a) and not in "Nova Genera et Species Plantarum" by the same author (Martius 1824b). In the databases "Tropicos" (Missouri Botanical Garden 2015) and "World Checklist of Selected Plant Families" (WCSP 2015), this information is conflicting. Aspidosperma pyrifolium occurs in Bolivia, Brazil and Paraguay (Missouri Botanical Garden 2015). In the state of Pará, this species was found only in Baixo Amazonas Mesoregion (IBGE 1990). According to Marcondes-Ferreira \& Kinoshita (1996), Aspidosperma pyrifolium belongs to section Aspidosperma. This species shares morphological features with Aspidosperma multiflorum, mainly fruit morphology, but differs from $A$. multiflorum by the chartaceous leaves, larger flowers (14-23 mm long), ovate corolla lobes and externally tomentose calyx.

15. Aspidosperma rigidum Rusby, Mem. New York Bot. Gard. 7: 323. 1927. (Figure 5k-o).

Trees 15-20 m high; trunk tortuous. Branches cylindrical, not suberous, sparsely lenticellate, glabrescent to glabrous, without cataphylls; latex whitish. Leaves alternate, arranged along the branches; petioles 1.7-2 cm long, glabrous; blade 6.1-9 x 3.7-5.5 $\mathrm{cm}$, chartaceous to coriaceous, flat, elliptic, apex acuminate, base attenuate or oblique, margin straight or revolute, concolorous, venation eucamptodromous, adaxial surface dull or lustrous, dark brown, glabrous, primary vein flat, secondary veins impressed, tertiary veins inconspicuous, abaxial surface dull, dark brown, glabrous, primary vein prominent, secondary veins prominulous, 12-15 pairs, tertiary veins inconspicuous. Inflorescences $4-5 \mathrm{~cm}$ long, axillary or terminal, corymbiform dichasia, tomentose. Flower buds with corolla lobes not twisted. Flowers $10-13 \mathrm{~mm}$ long; pedicellate, pedicel $2.7-4.2 \times 0.2-0.5 \mathrm{~mm}$, tomentose. Calyx $1.2-1.5 \times 1.5 \mathrm{~mm}$, tomentose externally, glabrous internally; lobes 5, 1-1.2 x 0.5-1 mm, ovate, apex acute. Corolla 4-8 x 1-1.2 mm, salverform, yellow, glabrous externally, pubescent along the tube internally; lobes $2-5 \times 1 \mathrm{~mm}$, erect or deflexed, lanceolate, apex acute. Stamens 1.6-3 mm long; filaments 1-2.2 $\mathrm{mm}$ long, pubescent; anthers 0.6-0.8 mm long, ovate, apex acute. Carpels $1.5 \mathrm{~mm}$ long; ovary $0.5 \times 0.5 \mathrm{~mm}$, globoid, glabrous; style $0.7 \mathrm{~mm}$ long; style-head $0.3 \mathrm{~mm}$ long, main body globose, with 2 oblong apical appendages. Follicles 5-7.5 x 3.9-7.8 cm, suborbicular, smooth, sessile or stipitate, not mucronate, lenticels inconspicuous, black, glabrous. Seeds 6-7 cm diam. orbicular; seminal nucleus central, without radial lines, nucleus $1.5 \mathrm{~cm}$ diam.

Material examined: BRAZIL, PARÁ: Alenquer, mata de Bom Futuro, 25.II.1953, fr., R.L. Fróes 29400 (IAN); Alenquer, Rio Curuá, estrada Urapurú-Água Branca, área de castanheira, 4. III.1953, fr., R.L. Fróes 29472 (IAN); Breu Branco, Rio Tocantins near Igarapé Cajazeirinha, approx. $30 \mathrm{~km} \mathrm{~N}$ of Itupiranga, 29. XI.1981, fl., D.C. Daly et al. 1610 (INPA, MG); Itaituba, árvore n ${ }^{\circ}$ 76-2-6, XII.1972, fl., J.M. Pires 13822 (IAN); Monte Alegre, Colônia Japonesa Assaizal, 22.IX.1953, fl., R.L. Fróes 30320 (IAN); Monte Alegre, Colônia Japonesa Assaizal, 22.IX.1953, fr., R.L. Fróes 30306 (IAN); Oriximiná, Comunidade Jauari, casa Irineu, 21.II.2010, fl., D.R. Oliveira et al. 216 (UEC); Parauapebas, Serra dos Carajás, Rio Parauapebas, control point at entrance to Serra Norte, ca. $39 \mathrm{~km}$ east of AMZA camp N-5, 23. VI.1982, fr., C.R. Sperling et al. 6318 (MG); Vitória do Xingu, CBVL, 21.II.2013, fl., T.C.S. Silva PSACF 960 (MG); Xinguara, Rio Vermelho, região do Tocantins, ao longo da margem do rio, 23.IV.1951, fr., R.L. Fróes 26988 (IAN, USP-scanned image).

Additional material: BRAZIL, AMAZONAS: Boca do Solimões, Paraná do Careiro, 7.VI.1948-2.VIII.1948, fl., A. Ducke 2132 (IAC, IAN, R); RONDÔNIA: Rio Jaru, BR-29, 21.IX.1962, fl., A.P. Duarte 7017 (INPA). PERU, HUÁNUCO: Pachitea, Codo de Pozuzo, trail S of settlement toward main river, $9^{\circ} 40^{\prime} \mathrm{S}, 7^{\circ} 25^{\prime} \mathrm{W}$, 18.X.1982, fl., R.B. Foster 9274 (F-scanned image).

Phytoecological region and phenology: Dense Ombrophilous Forest (terra firme forest). Flowers and fruits collected from June to April.

Common names: carapanaúba, carapanaúba-do-baixio.

Aspidosperma rigidum occurs in Bolivia, Brazil, Colombia, Costa Rica, Ecuador, Peru and Venezuela (Missouri Botanical Garden 2015). In the state of Pará, this species was found in Baixo Amazonas, Sudeste Paraense and Sudoeste Paraense Mesoregions (IBGE 1990). According to Marcondes-Ferreira \& Kinoshita (1996), Aspidosperma rigidum belongs to monospecific section Rigida. Aspidosperma rigidum can be distinguished from the other species occurring in Pará by the elliptic leaves with acuminate apex and the non-mucronate black follicles with inconspicuous lenticels.

16. Aspidosperma salgadense Markgr., Notizbl. Bot. Gart. Berlin-Dahlem 12(115): 554. 1935. (Figure 5p-s).

Trees 8-25 m high; trunk tortuous. Branches angular, not suberous, sparsely lenticellate, pubescent to glabrous, without cataphylls; latex whitish. Leaves subopposite or alternate, arranged along the branches; petioles 1-2 cm long, tomentose; blade $5.5-12.5 \times 3.5-4.8 \mathrm{~cm}$, chartaceous, flat, oblong or ovate, apex acute, base revolute, margin revolute, discolorous, venation brochidodromous, with marginal vein $1 \mathrm{~mm}$ from the margin, adaxial surface dull or lustrous, dark brown, glabrous, primary vein flat, secondary veins prominent, tertiary veins inconspicuous, abaxial surface dull, light brown, tomentose along the primary vein, primary vein prominent, secondary veins prominent, 20-22 pairs, tertiary veins inconspicuous. Inflorescences 5.6-6 cm long, terminal, corymbiform dichasia, tomentose. Flower buds with corolla lobes not twisted. Flowers 6.5-7.5 mm long; pedicellate, pedicel 1.7-2.2 x $1 \mathrm{~mm}$, tomentose. Calyx 2.5-2.7 x 2-2.5 mm, tomentose externally, pubescent at base and/ 

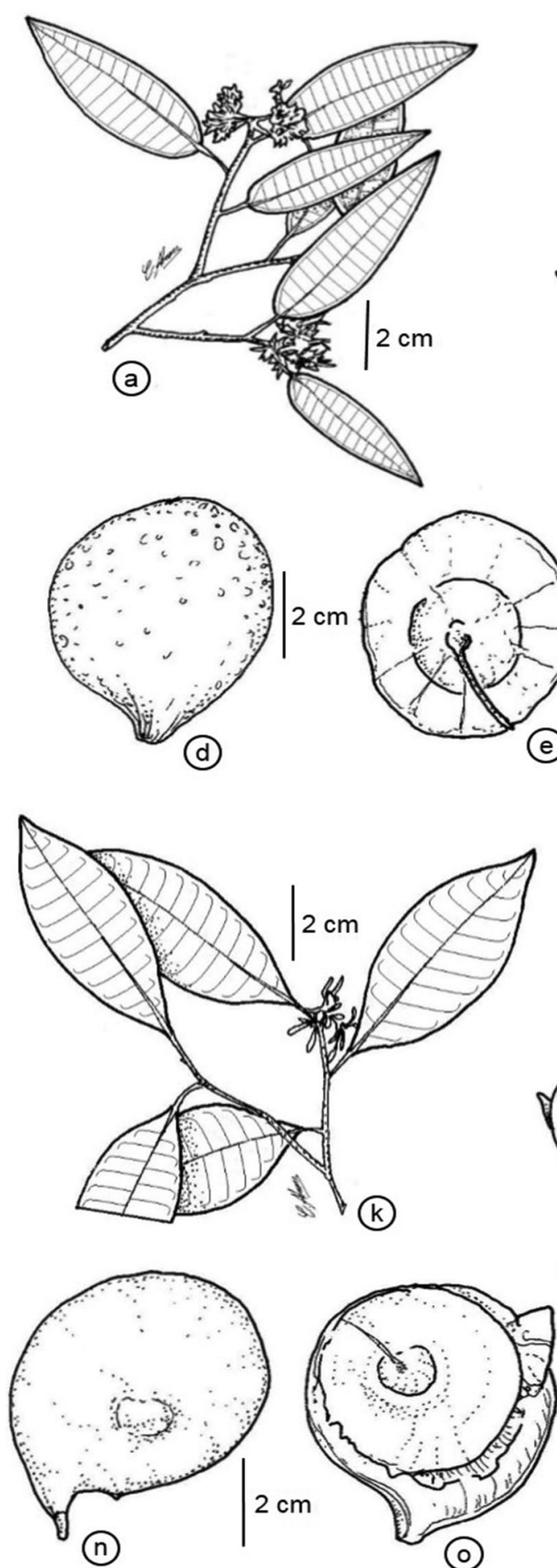

(n)
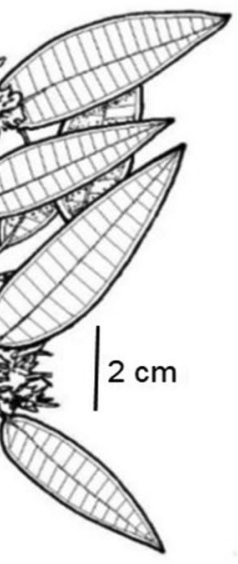

$2 \mathrm{~cm}$

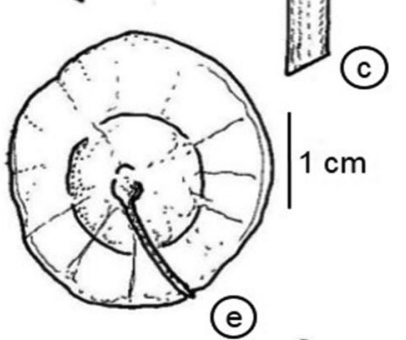

(e)
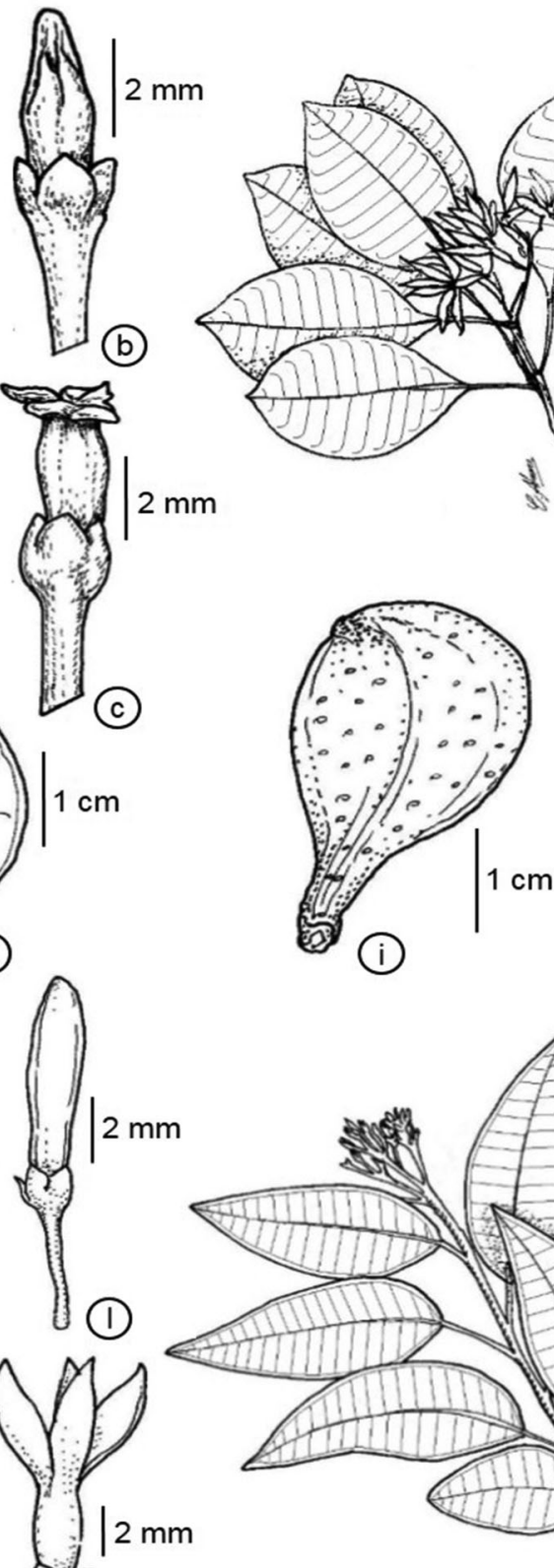

$2 \mathrm{~mm}$
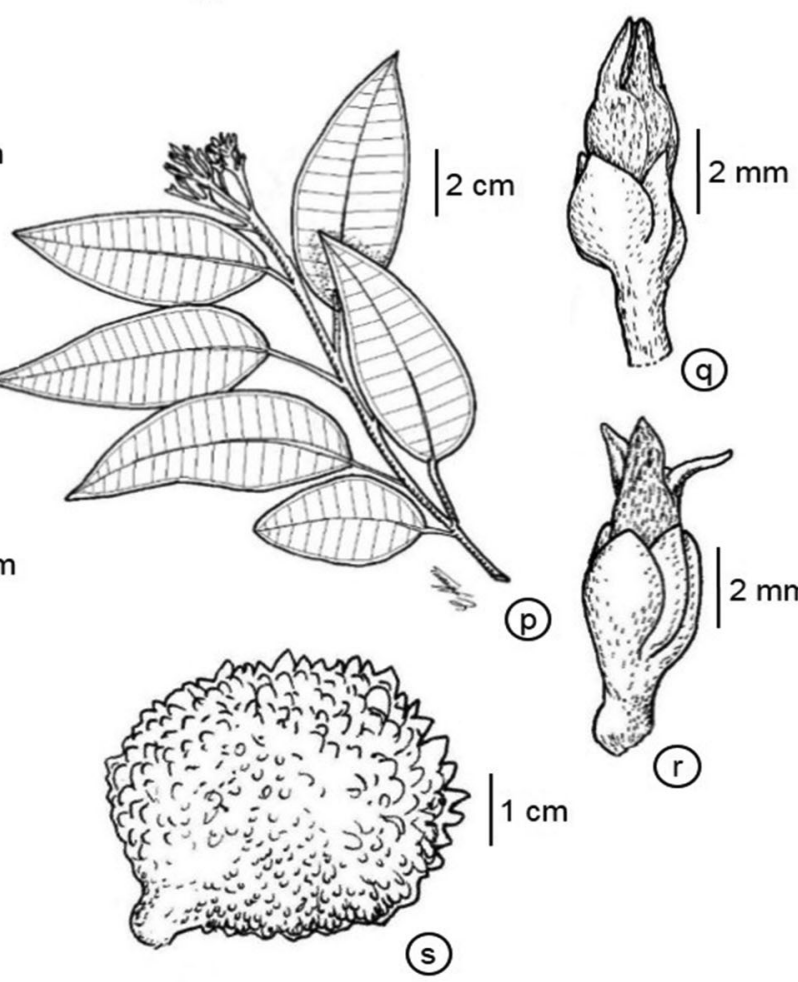

(a)

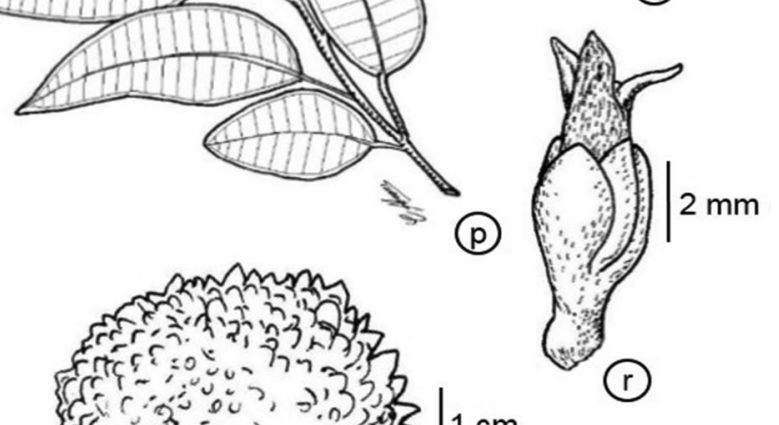

$2 \mathrm{~cm}$

Figure 5. a-e. Aspidosperma oblongum. f-j. A. pyrifolium. k-o. A. rigidum. p-s. A. salgadense: a, f, k, p. flowering branch; b, g, l, q. flower bud; c, h, m, r. flower; d, i, n, s. follicle; e, j, o. seed. a-e. Martin s.n. (P 4205125); N.T. Silva 1285; N.T. Silva 1855; R. Benoist 963. f-j. A. Rapini \& L.P. Queiroz 1156; L.P. Queiroz et al. 7261; M.G. Silva \& J. Maria 3357. k-o. A. Ducke 2132; R.B. Foster 9274; R.L. Fróes 26988. p-s. L.C. Antônio PSACF 266; R.L. Fróes 30286.

Figura 5. a-e. Aspidosperma oblongum. f-j. A. pyrifolium. k-o. A. rigidum. p-s. A. salgadense: a, f, k, p. ramo florido; b, g, l, q. botão floral; c, h, m, r. flor; d, i, n, s. folículo; e, j, o. semente. a-e. Martin s.n. (P 4205125); N.T. Silva 1285; N.T. Silva 1855; R. Benoist 963. f-j. A. Rapini \& L.P. Queiroz 1156; L.P. Queiroz et al. 7261; M.G. Silva \& J. Maria 3357. k-o. A. Ducke 2132; R.B. Foster 9274; R.L. Fróes 26988. p-s. L.C. Antônio PSACF 266; R.L. Fróes 30286. 
or at apex internally; lobes $5,1.5-2 \times 1 \mathrm{~mm}$, ovate, apex acuminate or acute. Corolla 3.5-5.5 x $1.5 \mathrm{~mm}$, tubular, white, tomentose externally, tomentose along the tube internally; lobes $1.5-2 \times 0.3$ $\mathrm{mm}$, patent, ovate, apex acute. Stamens $2-2.8 \mathrm{~mm}$ long; filaments 1-1.8 mm long, tomentose; anthers $1 \mathrm{~mm}$ long, lanceolate, apex acute. Carpels $1 \mathrm{~mm}$ long; ovary $0.5 \times 0.7 \mathrm{~mm}$, globoid, glabrous; style $0.2 \mathrm{~mm}$ long; style-head $0.3 \mathrm{~mm}$ long, main body oblong, with 2 oblong apical appendages. Follicles 4.3-6 x 3.2-5 cm, dolabriform, spinescent, stipitate, not mucronate, lenticels inconspicuous, brown, pubescent. Seeds not observed.

Material examined: BRAZIL, PARÁ: Aveiro, região do Tapajós, Boa Vista, 24.IX.1932, f1., Capucho 457 (IAN); Itaituba, árvore $n^{\circ}$ 38-4-25, XII.1972, st., J.M. Pires 13821 (IAN); Marabá, IX.1970, fl., J.M. Pires \& R.P. Belem 12644 (IAN); Monte Alegre, Rio Maicurú, 3:30 hrs por canoa de motor de poupa mais 1:30 hrs de caminhada acima da pista de pouso do Lageiro, 30.VII.1981, st., J. Jangoux \& B.G.S. Ribeiro 1575 (MG); Monte Alegre, Rio Maicurú, caminho de Caá-ussú à localidade Balança, 16.IX.1953, fl., R.L. Fróes 30286 (IAN); Monte Alegre, Rio Maicurú, entre Caá-ussú e localidade Balança, 15.IX.1953, fl., R.L. Fróes 30271 (IAN, NYscanned image, R); Santarém, Rio Curuá-Una, região do Planalto de Santarém, onde foi feito o levantamento estatístico florestal pelo IAN, SPVEA e FAO, 14.IX.1954, fl., R.L. Fróes 31382 (IAN); Vitória do Xingu, Travessão 55, 26.V.2012, fr., L.C. Antônio PSACF 266 (MG); Vitória do Xingu, Balança-km 9,5, 1. II.2013, fl., E. Takanohashi PSACF 919 (MG).

Phytoecological region and phenology: Dense Ombrophilous Forest (terra firme forest). Flowers and fruits collected from May to September.

Common names: carapanaúba.

According to Woodson (1951), Aspidosperma salgadense is a species endemic to Brazil, and occurs only in the state of Pará in Baixo Amazonas, Sudeste Paraense and Sudoeste Paraense Mesoregions (IBGE 1990, Missouri Botanical Garden 2015). Marcondes-Ferreira (1988) reduced Aspidosperma salgadense to be synonymous with $A$. discolor (section Excelsa), but we recognize $A$. salgadense as an accepted species due to observed differences between the specimens of the two species. Aspidosperma salgadense resembles $A$. discolor, especially flower structures, but differs from $A$. discolor by the angular branches and, mainly by the glabrous ovary, a feature that has already been highlighted by Markgraf (1935) in the protologue of A. salgadense.

17. Aspidosperma sandwithianum Markgr., Notizbl. Bot. Gart. Berlin-Dahlem 12(115): 561. 1935. (Figure 6a-e).

Trees 12-40 m high; trunk straight. Branches cylindrical, suberous, sparsely lenticellate, velutinous to glabrous, without cataphylls; latex orange to reddish. Leaves alternate, arranged along the branches; petioles $1-3 \mathrm{~cm}$ long, tomentose; blade 10.1-37.5 x 3-9.8 cm, coriaceous, bullate, oblong or obovate, apex acute, obtuse or retuse, base cuneate or oblique, margin revolute, discolorous, venation craspedodromous, adaxial surface dull, olive green or brown, tomentose along the primary vein, primary vein flat, secondary veins impressed, tertiary veins inconspicuous, abaxial surface dull, white or yellow, tomentose, primary vein prominent, secondary veins prominent, 26-34 pairs, tertiary veins inconspicuous. Inflorescences $6.5-15 \mathrm{~cm}$ long, axillary or terminal, corymbiform dichasia, tomentose. Flower buds with corolla lobes twisted. Flowers 6-8 mm long; pedicellate, pedicel 1-1.5 × $1 \mathrm{~mm}$, tomentose. Calyx 2-2.5 × 2$2.2 \mathrm{~mm}$, tomentose externally, pubescent at apex internally; lobes
5, 1.6-2 x $1 \mathrm{~mm}$, ovate, apex acute or obtuse. Corolla 4.5-6.2 x 1.2 $\mathrm{mm}$, salverform, yellow, glabrous externally, tomentose below the anthers internally; lobes $2.5-3.2 \times 0.3 \mathrm{~mm}$, erect, filiform, apex acute. Stamens $2 \mathrm{~mm}$ long; filaments $1.5 \mathrm{~mm}$ long, tomentose; anthers $0.5 \mathrm{~mm}$ long, ovate, apex apiculate or acute. Carpels $1.3 \mathrm{~mm}$ long; ovary $0.3 \times 0.3 \mathrm{~mm}$, globoid, glabrous; style $0.8 \mathrm{~mm}$ long; style-head $0.2 \mathrm{~mm}$ long, main body globose, with 2 oblong apical appendages. Follicles 10-12 x 8.5-10 cm, suborbicular, sulcate, stipitate, mucronate, lenticels inconspicuous, brown, velutinous. Seeds 6-7 cm diam., orbicular; seminal nucleus lateral, without radial lines, nucleus $2-2.5 \mathrm{~cm}$ diam.

Material examined: BRAZIL, PARÁ: Almeirim, Monte Dourado, Cova da Onça, $0^{\circ} 45^{\prime} \mathrm{S}, 52^{\circ} 45^{\prime} \mathrm{W}, 15$.VIII.1979, fl., N. T. Silva 5259 (MG); Almeirim, região do Jarí, estrada entre Tinguelim e Braço, 29.V.1970, fl., N.T. Silva 3172 (IAN); Almeirim, região do Jarí, estrada entre Planalto A e Tinguelin, km 21, 24.XI.1969, st., N.T. Silva 2855 (IAN); Belém, IPEAN, Reserva Mocambo, L-8-14, árvore n 95, 5.VIII.1968, f1., J.M. Pires \& N.T. Silva 11907 (IAN); Belém, IPEAN, Reserva Mocambo, L-8-14, árvore n 95, 17.VII.1968, fl., J.M. Pires \& N. T. Silva 11892 (IAN); Belém, Mocambo, Embrapa Forest Reserve, ca. $10 \mathrm{~km}$ from Belém, transect 2, $1^{\circ} 30^{\prime} \mathrm{S}, 47^{\circ} 59^{\prime} \mathrm{W}$, 13.XI.1984, st., A. Gentry 48924 (IAN); Belém, Reserva Mocambo (EMBRAPA), árvore 18-16-8, 11.I.1983, st., R. Lima \& R.P. Bahia 7 (MG); Belterra, 6.VIII.1947, st., G.A. Black 47-1150 (IAC); Breves, perto do Igarapé Arapijó, transecto para inventário florestal, Q 4-53, 7-30.VII.1956, st., J.M. Pires et al. 5060 (IAN); Marabá, Rio Itacaiunas, afl. do Rio Tocantins, Serra Buritirama (B 4), região com minério de manganês, árvore 7.9.3, VIII.1970, st., J.M. Pires \& R.P. Belem 12699 (IAN); Melgaço, Floresta Nacional de Caxiuanã, local onde será construída a Estação Científica "Ferreira Penna", 2-15.II.1991, st., A.S.L. da Silva et al. 2350 (MG); Moju, 25.VIII.1975, st., N. T. Silva 3961 (IAN).

Additional material: BRAZIL, RORAIMA: Paracaima, as proximidades da divisa com a Venezuela, $\mathrm{km} \mathrm{11-2} \mathrm{do}$ marco BV-9, Cordilheira do Paracaima, 19.IX.1979, fr., N.A. Rosa \& O.C. Nascimento 3503 (MG). FRENCH GUIANA, RÉMIRE-MONTJOLY: Haut Oyapock, Mt. St. Marcel, layon camp Couleuvre-Mt. St. Marcel, 27.III.1976, fr., C. Sastre 4564 (P-scanned image).

Phytoecological region and phenology: Dense Ombrophilous Forest (terra firme forest). Flowers and fruits collected from May to September.

Common names: araracanga, araracanga-branca, cabeça-dearara, canoinha.

Aspidosperma sandwithianum occurs in Brazil, French Guiana, Guyana and Suriname (Missouri Botanical Garden 2015). In the state of Pará, this species was found in Baixo Amazonas, Marajó, Metropolitana de Belém, Nordeste Paraense and Sudeste Paraense Mesoregions (IBGE 1990). In "Livro Vermelho da Flora do Brasil" (Rapini et al. 2013), Aspidosperma sandwithianum was assigned to the Data Deficient category (DD), but in "Lista de Espécies da Flora e da Fauna Ameaçadas no Estado do Pará" (Pará 2007), it was listed as a Vulnerable species (VU). Marcondes-Ferreira (1988) reduced Aspidosperma sandwithianum to be synonymous with $A$. spruceanum (section Nobilia), but we found both taxa to have differences significant enough to be recognized as distinct species. Therefore, Aspidosperma sandwithianum is accepted here at the species level. Aspidosperma sandwithianum resembles $A$. spruceanum, mainly flower features, but differs from $A$. spruceanum by the bullate 
leaves, tomentose primary vein and impressed secondary veins on the adaxial surface, and suborbicular follicles.

18. Aspidosperma schultesii Woodson, Ann. Missouri Bot. Gard. 38(2): 168. 1951. (Figure 6f-j).

Trees 12-30 m high; trunk tortuous. Branches cylindrical, suberous, sparsely lenticellate, glabrescent to glabrous, without cataphylls; latex whitish. Leaves alternate, arranged along the branches; petioles $2.5-4.3 \mathrm{~cm}$ long, glabrescent to glabrous; blade $7.6-15$ x $3.1-7 \mathrm{~cm}$, chartaceous, flat, elliptic or obovate, apex acute, obtuse or retuse, base cuneate, attenuate or oblique, margin revolute, discolorous, venation craspedodromous, adaxial surface dull, dark brown, glabrous, primary vein prominulous, secondary veins impressed, tertiary veins inconspicuous, abaxial surface dull, light brown, pubescent to glabrous, primary vein prominent, secondary veins impressed, 18-20 pairs, tertiary veins inconspicuous. Inflorescences 9-11 cm long, terminal, corymbiform dichasia, tomentose. Flower buds with corolla lobes twisted. Flowers 11-20 mm long; pedicellate, pedicel $1.5-4$ x $0.5 \mathrm{~mm}$, tomentose. Calyx 1.5$2 \times 1.5-2 \mathrm{~mm}$, tomentose externally, glabrous internally; lobes 5, 1$1.5 \times 1 \mathrm{~mm}$, ovate, apex acute. Corolla 13-15 × 1.5-2 mm, salverform, yellow, pubescent externally, pubescent along the corolla internally; lobes 6-10 x 1.5-3 mm, patent, lanceolate, apex acute. Stamens 1.7-3 mm long; filaments 1-2 mm long, tomentose; anthers $0.7-1 \mathrm{~mm}$ long, ovate, apex acute. Carpels $1.5 \mathrm{~mm}$ long; ovary 0.7 x $0.8 \mathrm{~mm}$, globoid, tomentose; style $0.5 \mathrm{~mm}$ long; stylehead $0.3 \mathrm{~mm}$ long, main body globose, with 2 oblong apical appendages. Follicles 8-12.3 x 6-9.4 cm, suborbicular, smooth or sulcate, stipitate, mucronate, lenticels inconspicuous, black, glabrous. Seeds $6-7.8 \mathrm{~cm}$ diam. orbicular; seminal nucleus central, with radial lines, nucleus $2.5 \mathrm{~cm}$ diam.

Material examined: BRAZIL, PARÁ: Almeirim, Monte Dourado, estrada Perimetral (COMARA), 19.VI.1984, fl., N.T. Silva 5375 (INPA, MG); Jacareacanga, Rio São Manoel, posto do índios Caiabí, 7.I.1952, fr., J.M. Pires 3860 (IAN); Novo Progresso, Serra do Cachimbo, BR-163, Cuiabá-Santarém highway, km 872, 6.XI.1977, st., G.T. Prance et al. P24968 (MG, NYscanned image).

Additional material: BRAZIL, AMAZONAS: Apuí, floresta na beira do Rio Sucunduri acampamento, 26.VI.2006, fl., C. E. Zartman et al. 5562 (INPA); Carauari, Juruá I, VII.1980, st., A.S.L. Silva et al. 908 (MG); Manaus, Reserva Florestal Ducke, Manaus-Itacoatiara, km 26, 9.III.1994, fl., A. Vicentini \& C.F. Silva 404 (MG); Manaus, Reserva Florestal Ducke, Manaus-Itacoatiara, km 26, 1.VIII.1995, st., A.A. Oliveira \& P. A.C.L. Assunção 2807 (IAN); Tefé, estrada do Luc 3, Porto Urucú, 16.VII.1991, fl., A.S. Tavares et al. 414 (INPA); MATO GROSSO: Aripuanã, km 245 da BR-174, projeto Juina, linha 4, 19.I.1979, fr., M.G. Silva \& A. Pinheiro 4323 (MG); Vila Bela da Santíssima Trindade, $58 \mathrm{~km} \mathrm{~S}$ of Rondônia state line on BR-364 from Vilhena to Cáceres, 2.XI.1985, fr., W. Thomas et al. 4775 (INPA); RONDÔNIA: Road Vilhena to Colorado, $28 \mathrm{~km}$ from Vilhena (20 km from BR-364), forest at Rio Vermelho, 28.X.1979, fr., J.L. Zarucchi et al. 2814 (INPA).

Phytoecological region and phenology: Dense Ombrophilous Forest (terra firme forest). Flowers and fruits collected from January to November.

Common names: araracanga-rana.

Aspidosperma schultesii occurs in Brazil, French Guiana, Peru and Venezuela (Missouri Botanical Garden 2015). In the state of Pará, this species was found in Baixo Amazonas and Sudoeste Paraense Mesoregions (IBGE 1990). According to Marcondes-Ferreira \& Kinoshita (1996), Aspidosperma schultesii belongs to monospecific section Schultesia. Aspidosperma schultesii was one of the most easily recognized species, and can be differentiated from the other species occurring in Pará by the larger flowers (11-20 mm long), mucronate black follicles with inconspicuous lenticels and seminal nucleus with radial lines. Aspidosperma schultesii is the only species from the state with this last feature.

19. Aspidosperma spruceanum Benth. ex Müll.Arg. in C.F.P. von Martius \& auct. suc. (eds.), Fl. Bras. 6(1): 52. 1860. (Figure $6 \mathrm{k}-\mathrm{o})$.

Trees 6-38 m high; trunk straight. Branches cylindrical, suberous, sparsely lenticellate, tomentose to glabrescent, without cataphylls; latex reddish. Leaves alternate, arranged along the branches; petioles 1-2.9 cm long, tomentose to glabrescent; blade 7.2-16.2 x 3.1-6.1 cm, coriaceous, flat, oblong or obovate, apex acute or obtuse, base cuneate or oblique, margin revolute, discolorous, venation craspedodromous, adaxial surface lustrous, olive green or brown, glabrous, primary vein flat or prominulous, secondary veins prominulous, tertiary veins conspicuous, abaxial surface dull, white, tomentose to glabrous, primary vein prominent, secondary veins prominulous, 25-28 pairs, tertiary veins conspicuous or inconspicuous. Inflorescences $8.5-10 \mathrm{~cm}$ long, terminal, corymbiform dichasia, tomentose. Flower buds with corolla lobes twisted. Flowers $8.5-9 \mathrm{~mm}$ long; pedicellate, pedicel 1.7-2 x 0.5-0.7 mm, tomentose. Calyx 2-2.5 x 2-3 mm, tomentose externally, pubescent at apex internally; lobes 5, 1.5-1.7 x $1 \mathrm{~mm}$, ovate, apex acute. Corolla 6.5-7 x $1.5 \mathrm{~mm}$, salverform, yellow, glabrous externally, tomentose below the anthers internally; lobes $3.5-4$ x $0.3 \mathrm{~mm}$, erect, filiform, apex acute. Stamens 2.5-3 mm long; filaments 2-2.5 mm long, tomentose; anthers 0.5 $\mathrm{mm}$ long, ovate, apex acute. Carpels 1.4-2.4 mm long; ovary $0.3 \mathrm{x}$ $0.5 \mathrm{~mm}$, globoid, glabrous; style 0.7-1.7 mm long; style-head 0.4 $\mathrm{mm}$ long, main body globose, with 2 oblong apical appendages. Follicles 10.1-14 x 6-10.1 cm, dolabriform, sulcate, stipitate, mucronate or not, lenticels inconspicuous, brown, velutinous to glabrescent. Seeds 5.8-6.6 cm diam., orbicular; seminal nucleus lateral, without radial lines, nucleus $1.6-3.2 \mathrm{~cm}$ diam.

Material examined: BRAZIL, PARÁ: Almeirim, região do Rio Jarí, Monte Dourado, Planalto A, 9.IX.1968, fl., N.T. Silva 918 (IAN, NY-scanned image); Oriximiná, Porto Trombetas, próximo a área industrial, 9.X.1986, fl., E. Soares 221 (INPA); Porto de Moz, R. Xingu, margem esquerda do rio, região onde foi feito um levantamento estatístico florestal pelo IAN, SPVEA e FAO, 18.IX.1955, fr., R.L. Fróes 32377 (IAN); Santarém, Reserva Florestal de Curuá-Una, Planalto Alto II a 1,5 km do Flanco, 7.X.1963, fr., Tressel 22 (INPA); Santarém, região do Rio Curuá-Una, Baixo Amazonas, VI.1957, fl., R.L. Fróes 33893 (IAN); Tucuruí, estrada de ferro Santa Rosa, 1. VI.1981, fr., L.Coêlho, 1843 (INPA).

Additional material: BRAZIL, AMAZONAS: Barcelos, ilha periodicamente inundável, 8.IX.1962, st., A.P. Duarte 7007 (RB); Manaus, Reserva Florestal Ducke, próximo ao Igarapé Sempre Viva, 26.IX.1957, fr., E. Ferreira 109-57 (INPA); Manaus, Rio Tarumã-mirim, 19.X.1943, fr., A. Ducke 1419 (R); Maués, along Rio Apoquitaua, just above mouth of Rio Pacoval, 27.VII.1983, fr., J.L. Zarucchi 3210 (INPA); Rio Negro, boca do Rio Branco, 2.VII.1976, fl., L.F. Coêlho 538 (MG); Santa Isabel do Rio Negro, margem inundada do rio, 9.VI.1937, st., A. Ducke 511 (R); Santa Isabel do Rio Negro, Tapurucuara, Rio Negro, beira do rio, 7.II.1959, fr., P. Cavalcante 547 (MG); MARANHÃO: São Luiz, Sítio Tambaú, transectum 2-2-11, 1.VIII.1980, st., M.G. Silva 5594 (MG); RIO DE JANEIRO: 
Pereira, A.S.S. et al.

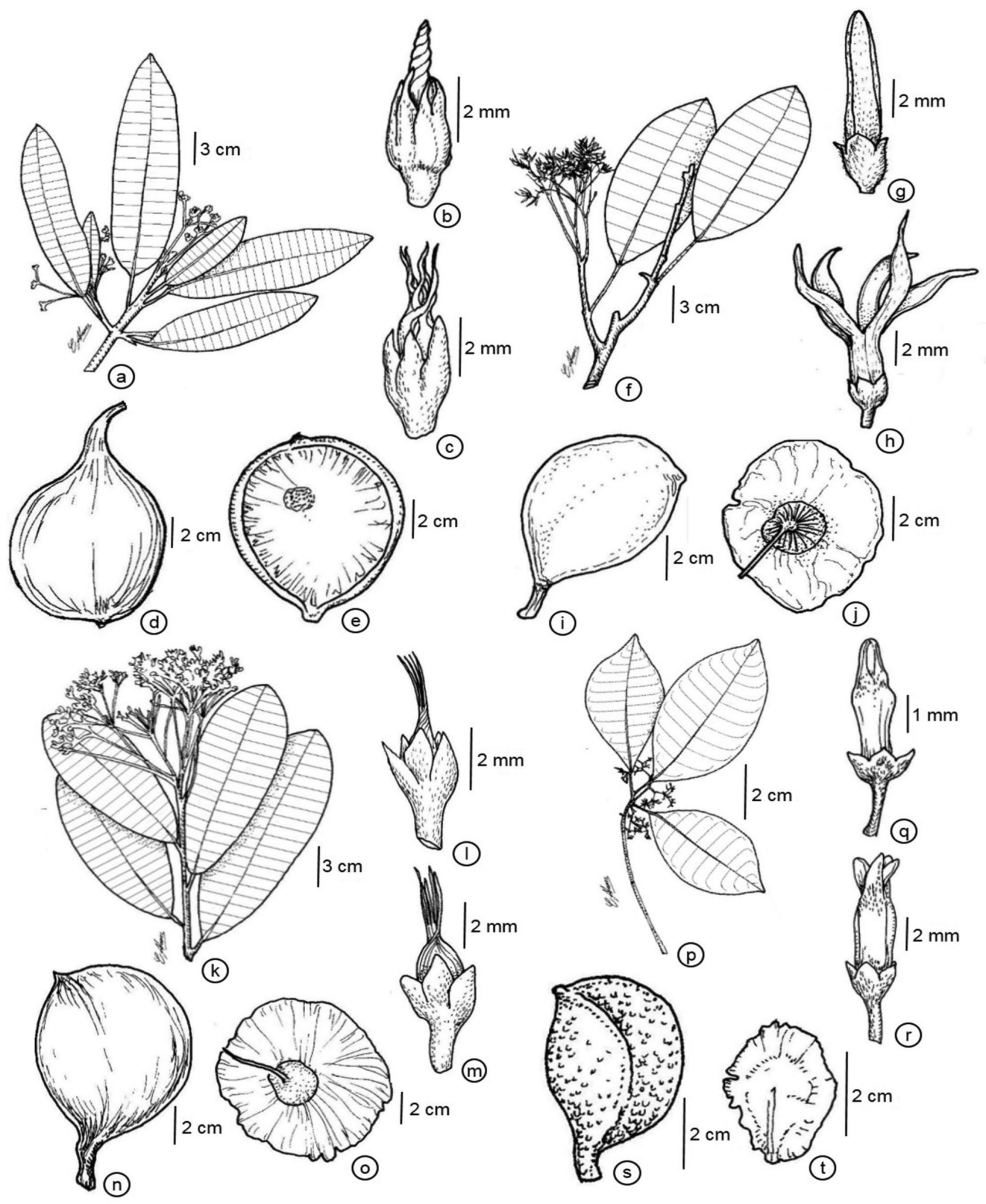

Figure 6. a-e. Aspidosperma sandwithianum. f-j. A. schultesii. k-o. A. spruceanum. p-t. A. subincanum: a, f, k, p. flowering branch; b, g, l, q. flower bud; c, h, m, r. flower; d, i, n, s. follicle; e, j, o, t. seed. a-e. C. Sastre 4564; J.M. Pires \& N.T. Silva 11907. f-j. J.L. Zarucchi et al. 2814; N.T. Silva 5375; W. Thomas et al. 4775. k-o. E. Ferreira 109-57; J.G. Kuhlmann s.n. (P 4204499); L.F. Coêlho 538. p-t. A.T.G. Dias 398; C.F.P. von Martius 262; G.T. Prance et al. 9494.

Figura 6. a-e. Aspidosperma sandwithianum. f-j. A. schultesii. k-o. A. spruceanum. p-t. A. subincanum: a, f, k, p. ramo florido; b, g, 1, q. botão floral; c, h, m, r. flor; d, i, n, s. folículo; e, j, o, t. semente. a-e. C. Sastre 4564; J.M. Pires \& N.T. Silva 11907. f-j. J.L. Zarucchi et al. 2814; N.T. Silva 5375; W. Thomas et al. 4775. k-o. E. Ferreira 109-57; J.G. Kuhlmann s.n. (P 4204499); L.F. Coêlho 538. p-t. A.T.G. Dias 398; C.F.P. von Martius 262; G.T. Prance et al. 9494 
Sumaré, 6.VII.1926, fr., J.G. Kuhlmann s.n. (P 4204499-scanned image).

Phytoecological region and phenology: Dense Ombrophilous Forest (terra firme forest). Flowers and fruits collected from February to October.

Common names: araracanga, araracanga-amarela, pau-amarelo, piquiá-marfim, piquiá-marfim-do-branco.

Aspidosperma spruceanum is a widely distributed species, occurring in Bolivia, Brazil, Colombia, Costa Rica, Ecuador, French Guiana, Guatemala, Guyana, Honduras, Mexico, Nicaragua, Panama and Suriname (Missouri Botanical Garden 2015). In the state of Pará, this species was found in Baixo Amazonas and Sudeste Paraense Mesoregions (IBGE 1990). According to Marcondes-Ferreira \& Kinoshita (1996), Aspidosperma spruceanum belongs to section Nobilia. Among the species studied, Aspidosperma spruceanum was one of the most difficult to recognize, as it shares morphological features with both $A$. album and $A$. sandwithianum, especially flower morphology. However, Aspidosperma spruceanum differs from the former by the olive green or brown color of the leaf blade on the adaxial surface and from the latter by the flat leaves and glabrous primary vein on the adaxial surface. Additionally, Aspidosperma spruceanum is differentiated from both species by the prominulous secondary veins on the adaxial surface and dolabriform follicles. Aspidosperma spruceanum is most often confused with $A$. desmanthum, which was mentioned by Müller-Argoviensis (1860) in the protologue of A. spruceanum, and by Marcondes-Ferreira (1988), but can be distinguished from $A$. desmanthum by the prominulous secondary veins on both leaf surfaces.

20. Aspidosperma subincanum Mart., Flora 21(2): 82. 1838. (Figure 6p-t).

Trees 6-20 m high; trunk straight. Branches cylindrical, not suberous, densely lenticellate, glabrescent to glabrous, with cataphylls; latex whitish. Leaves alternate, congested at apex of branches; petioles $0.9-2 \mathrm{~cm}$ long, tomentose to glabrescent; blade 5.3-19.5 x 2.8-10.2 cm, chartaceous, flat, elliptic, apex acuminate or acute, base attenuate or oblique, margin straight, discolorous, venation eucamptodromous, adaxial surface dull, olive green, pubescent to glabrescent, primary vein flat, secondary veins prominent, tertiary veins inconspicuous, abaxial surface dull, light green, tomentose, primary vein prominent, secondary veins prominent, 17-22 pairs, tertiary veins conspicuous. Inflorescences 4-6 cm long, terminal, corymbiform dichasia, tomentose. Flower buds with corolla lobes not twisted. Flowers 5-7 mm long; pedicellate, pedicel 1.3-1.5 x 0.5 mm, tomentose. Calyx 1-1.2 x 1-1.5 $\mathrm{mm}$, tomentose externally, glabrous internally; lobes $5,1 \times 0.5 \mathrm{~mm}$, ovate, apex acute. Corolla 4-5.2 x $1 \mathrm{~mm}$, tubular, white, pubescent at apex externally, pubescent along the tube internally; lobes 1-1.5 x $0.7 \mathrm{~mm}$, patent, ovate, apex acute. Stamens 2-2.5 mm long; filaments 1.5-2 mm long, pubescent; anthers $0.5 \mathrm{~mm}$ long, ovate, apex apiculate or acute. Carpels $1.8 \mathrm{~mm}$ long; ovary $0.5 \times 0.5 \mathrm{~mm}$, ovoid, tomentose; style $1 \mathrm{~mm}$ long; style-head $0.3 \mathrm{~mm}$ long, main body oblong, with 2 filiform apical appendages. Follicles 4-7.5 x 2-3.8 cm, pyriform or suborbicular, smooth, stipitate, mucronate or not, lenticels conspicuous, brown, pubescent. Seeds $3 \times 5 \mathrm{~cm}$, ovate; seminal nucleus central, without radial lines, nucleus $2 \mathrm{~cm}$ diam.

Material examined: BRAZIL, PARÁ: Alenquer, Sete Varas airstrip on Rio Curuá, $0^{\circ} 95^{\prime} \mathrm{S}, 5^{\circ}{ }^{\circ} 92^{\prime} \mathrm{W}, 4$.VIII.1981, fr., J.J. Strudwick et al. 4045 (MG); Altamira, Rio Xingu, morro atrás do Lago do Muruci, 20.X.1986, fl., A.T.G. Dias 398 (MG); Canaã dos Carajás, 27.XII.2000-6.I.2001, fr., L.C.
B. Lobato et al. 2587 (MG); Monte Alegre, estrada de Caissú para a localidade Balança, 15.IX.1953, st., R.L. Fróes 30238 (IAN, INPA, R); Monte Alegre, Serra do Ererê, parte do Mirante, $1^{\circ} 55^{\prime}$ S, $54^{\circ} 15^{\prime} \mathrm{W}, 6$. XI.1987, f1., C.A.C. Ferreira 9503 (INPA, MG, RB); Monte Alegre, região do Igarapé da Formosa, 30.IX.1953, fr., R.L. Fróes 30450 (IAN); Óbidos, Flota de Trombetas, 058'10"S, 55³1'03”W, IV.2008, fr., L.C. B. Lobato \& C.A.S. Silva 3397 (MG); Pau D'Arco, Marajoara, 10.X.1997, f1., J. Grogan 24 (IAN, INPA, MG); São Geraldo do Araguaia, morro 3, 15.VI.1995, fr., M.N. Bastos \& M.R. Cordeiro 2173 (IAN, MG).

Additional material: BRAZIL, MARANHÃO: Presidente Dutra, roadside \& capoeira approx. $10 \mathrm{~km} \mathrm{E}$ of Barra do Corda, 10.X.1980, fl., D.C. Daly et al. D541 (MG); MATO GROSSO: Cuiabá, s.d., fr., C.F.P. von Martius 262 (Kscanned image); Chapada dos Guimarães, behind Refeitorio, about 1 km from Buriti, 26.X.1973, fl., G.T. Prance et al. 19422 (INPA); Drainage of the upper Rio Araguaia, Serra Azul, ca. $85 \mathrm{~km}$ S of Xavantina, 17.VI.1966, fr., H.S. Irwin et al. 17354 (RB); RORAIMA: Dormida, foothills of Serra da Lua, 25. I.1969, fr., G.T. Prance et al. 9494 (NY-scanned image).

Phytoecological region and phenology: Dense Ombrophilous Forest (terra firme forest) and Savannah. Flowers and fruits collected from April to November.

Common names: guatambu, pau-pereira, pereiro.

The information about the protologue of Aspidosperma subincanum was checked in its original publication. The guide "Taxonomic Literature" (Stafleu \& Cowan 1981), also states that Aspidosperma subincanum was first described in "Flora" by Martius (1838) and not in "Herbarium florae brasilienses" by the same author (Martius 1837-1841). In the database "Tropicos" (Missouri Botanical Garden 2015) this information is conflicting. Aspidosperma subincanum occurs in Bolivia and Brazil (Missouri Botanical Garden 2015). In the state of Pará, this species was found in Baixo Amazonas, Sudeste Paraense and Sudoeste Paraense Mesoregions (IBGE 1990). According to Marcondes-Ferreira \& Kinoshita (1996), Aspidosperma subincanum belongs to section Aspidosperma. Aspidosperma subincanum can be differentiated from the other species in section Aspidosperma occurring in Pará by the tubular corolla, tomentose ovary and non-twisted corolla lobes.

\section{Acknowledgments}

We are grateful to the Coordenação de Aperfeiçoamento de Pessoal de Nível Superior (CAPES, Office for the Advancement of Higher Education) for the master's grant provided to the first author, and the grant provided by the PNADB (Grant no. PNADB 922/2010). We also thank the Universidade Federal Rural da Amazônia (UFRA) and Museu Paraense Emílio Goeldi (MPEG) for the logistics support and infrastructure, and the curators and technicians from the visited herbaria for allowing us to study important collections of Aspidosperma.

\section{References}

APG III - THE ANGIOSPERM PHYLOGENY GROUP III. 2009. An update of the Angiosperm Phylogeny Group classification for the orders and families of flowering plants: APG III. Bot. J. Linn. Soc. 161(2):105-121, http://dx.doi.org/10.1111/j.1095-8339.2009. 00996.x 
BRASIL. 1999. O Estado do Pará. http://www.dc.mre.gov.br/imagense-textos/revista1-mat8.pdf (last accessed on 01/05/2015).

BRUMMITT, R.K. \& POWELL, C.E. 1992. Authors of plant names: a list of authors of scientific names of plants, with recommended standard forms of their names, including abbreviations. Royal Botanic Gardens, Kew.

DE CANDOLLE, A.L.P.P. 1844. Apocynaceae. In Prodomus Systematis Naturalis Regni Vegetabilis (A.P. de Candolle \& A.L.P.P. de Candolle, eds.). Sumptibus Sociorum Treuttel et Wuürtz, Paris, pars 8, p.317-489.

DUARTE, A.P. 1970. Contribuição para uma revisão do gênero Aspidosperma. An. Acad. Bras. Ciênc. 42:289-327.

ENDRESS, M.E. \& BRUYNS, P.V. 2000. A revised classification of the Apocynaceae s.l. Bot. Rev. 66(1):1-56, http://dx.doi.org/ 10.1007/BF02857781

ENDRESS, M.E., LIEDE-SCHUMANN, S. \& MEVE, U. 2007. Advances in Apocynaceae: the enlightenment, an introduction. Ann. Missouri Bot. Gard. 94(2):259-267, http://dx.doi.org/10.3417/ 0026-6493(2007)94[259:AIATEA]2.0.CO;2

ENDRESS, M.E., LIEDE-SCHUMANN, S. \& MEVE, U. 2014. An updated classification for Apocynaceae. Phytotaxa 159(3):175-194, http://dx.doi.org/10.11646/phytotaxa.159.3.2

FIDALGO, O. \& BONONI, V.L. 1984. Técnicas de coleta, preservação e herborização de material botânico. Instituto de Botânica, São Paulo.

GOMES, S.M. 2008. Morfo-anatomia de frutos secos em espécies de Apocynaceae: significado ecológico e evolutivo. Acta Bot. Bras. 22(2):521-534, http://dx.doi.org/10.1590/S0102-33062008000200019

GOMES, S.M. \& CAVALCANTI, T.B. 2001. Morfologia floral de Aspidosperma Mart. \& Zucc. (Apocynaceae). Acta Bot. Bras. 15(1): 73-88, http://dx.doi.org/10.1590/S0102-33062001000100009

IBGE - INSTITUTO BRASILEIRO DE GEOGRAFIA E ESTATÍSTICA. 1990. Divisão regional do Brasil em Mesorregiões e Microrregiões geográficas. IBGE, Rio de Janeiro.

IBGE - INSTITUTO BRASILEIRO DE GEOGRAFIA E ESTATÍSTICA. 2004. Mapa de Biomas do Brasil: primeira aproximação. Escala 1:5.000.000. IBGE, Rio de Janeiro.

IBGE - INSTITUTO BRASILEIRO DE GEOGRAFIA E ESTATÍSTICA. 2008. Estado do Pará: vegetação. Escala 1:1.800.000. IBGE, Rio de Janeiro.

ITHAKA. 2015. JSTOR Global Plants. http://plants.jstor.org/ (last accessed on 01/05/2015).

IUCN - INTERNATIONAL UNION FOR CONSERVATION OF NATURE AND NATURAL RESOURCES. 2015. The IUCN Red List of Threatened Species Version 2015.2. http://www.iucnredlist.org/ (last accessed on 26/06/2015).

JBRJ - INSTITUTO DE PESQUISA DO JARDIM BOTÂNICO DO RIO DE JANEIRO. 2015. Lista de Espécies da Flora do Brasil. http://floradobrasil.jbrj.gov.br/ (last accessed on 01/05/2015).

KINOSHITA, L.S. \& SIMÕES, A.O. 2005. Flora da Serra do Cipó, Minas Gerais: Apocynaceae s.str. (Rauvolfioideae e Apocynoideae). Bol. Bot. Univ. São Paulo 23(2):235-256, http://dx.doi. org/10.11606/issn.2316-9052.v23i2p235-256

KOCH, I., RAPINI, A., KINOSHITA, L.S., SIMÕES, A.O. \& SPINA, A.P. 2015. Apocynaceae. In Lista de Espécies da Flora do Brasil. Jardim Botânico do Rio de Janeiro. http://floradobrasil.jbrj.gov.br/ jabot/floradobrasil/FB4520 (last accessed on 01/05/2015).

LAWRENCE, G.H.M. 1973. Taxonomy of Vascular Plants. The Macmillan Press, New York.

LORENZI, H. 2008. Árvores brasileiras: manual de identificação e cultivo de plantas arbóreas nativas do Brasil. Vol. 1. Instituto Plantarum, Nova Odessa.

LORENZI, H. 2009. Árvores brasileiras: manual de identificação e cultivo de plantas arbóreas nativas do Brasil. Vol. 2. Instituto Plantarum, Nova Odessa.

MARCONDES-FERREIRA, W. 1988. Aspidosperma Mart., nom. cons. (Apocynaceae): estudos taxonômicos. Tese de doutorado, Universidade Estadual de Campinas, Campinas.
MARCONDES-FERREIRA, W. 1991. Novos taxa para o gênero Aspidosperma Mart. nom. cons. (Apocynaceae). Rev. Bras. Bot. 14 (2):127-132.

MARCONDES-FERREIRA, W. 1999. A new species of Aspidosperma Mart. (Apocynaceae) from Bahia, Brazil. Brittonia 51(1):74-76, http://dx.doi.org/10.2307/2666559

MARCONDES-FERREIRA, W. \& KINOSHITA, L.S. 1996. Uma nova divisão infragenérica para Aspidosperma Mart. (Apocynaceae). Rev. Bras. Bot. 19(2):203-214.

MARKGRAF, F. 1935. Neue Apocynaceen aus Südamerika VI. Notizbl. Bot. Gart. Berlin-Dahlem 12(115):553-561, http://dx.doi. org/10.2307/3994850.

MARTIUS, C.F.P. 1824a. Ankündigung der fortsetzung eines werkes über brasilianische pflanzen. Flora 7(1):129-142, http://dx.doi.org/ $10.3417 / 2009069$

MARTIUS, C.F.P. 1824b. Nova Genera et Species Plantarum. Vol.1. Typis Lindaueri, Munich.

MARTIUS, C.F.P. 1838. Martii Herbarium Florae Brasiliensis: continuatio. Flora 21(2):49-96.

MARTIUS, C.F.P. 1837-1841. Herbarium Florae Brasiliensis. S.n., Munich.

MATOZINHOS, C.N. \& KONNO, T.U.P. 2011. Diversidade taxonômica de Apocynaceae na Serra Negra, MG, Brasil. Hoehnea 38 (4):569-595.

MISSOURI BOTANICAL GARDEN. 2015. Tropicos. http://www. tropicos.org/ (last accessed on 01/05/2015).

MOROKAWA, R., SIMÕES, A.O. \& KINOSHITA, L.S. 2013. Apocynaceae s.str. do Parque Nacional da Serra da Canastra, Minas Gerais, Brasil. Rodriguésia 64(1):179-199, http://dx.doi.org/ $10.1590 / \mathrm{S} 2175-78602013000100015$

MÜLLER-ARGOVIENSIS, J. 1860. Apocynaceae. In Flora Brasiliensis (C.F.P. Martius, ed.). F. Fleischer, Leipzig, v.6, pars 1, p.1-196.

NAZAR, N., GOYDER, D.J., CLARKSON, J.J., MAHMOOD, T. \& CHASE, M.W. 2013. The taxonomy and systematics of Apocynaceae: where we stand in 2012. Bot. J. Linn. Soc. 171(3):482-490, http://dx.doi.org/10.1111/boj.12005

OLIVEIRA, V.B., FREITAS, M.S.M., MATHIAS, L., BRAZFILHO, R. \& VIEIRA, I.J.C. 2009. Atividade biológica e alcalóides indólicos do gênero Aspidosperma (Apocynaceae): uma revisão. Rev. Bras. Plantas Med. 11(1):92-99, http://dx.doi.org/ 10.1590/S1516-05722009000100015

PARÁ. 2007. Resolução n 54, de 24 de outubro de 2007, homologa a "Lista de Espécies da Flora e da Fauna Ameaçadas no Estado do Pará. http://www.sema.pa.gov.br/2009/03/27/9439/ (last accessed on $01 / 05 / 2015$ ).

PARÁ. 2010. O Pará. http://www.pa.gov.br/O_Para/opara.asp (last accessed on 01/05/2015).

PEREIRA, M.M., JÁCOME, R.L.R.P., ALCÂNTARA, A.F.C., ALVES, R.B. \& RASLAN, D.S. 2007. Alcalóides indólicos isolados de espécies do gênero Aspidosperma (Apocynaceae). Quim. Nova 30(4):970-983, http://dx.doi.org/10.1590/S0100-40422 007000400037

PICHON, M. 1947. Classification des Apocynacées VII: genre Aspidosperma. Bull. Mus. Natl. Hist. Nat., sér.2, 19(4):362-369, http:// dx.doi.org/10.1080/00378941.1947.1083457542.

RADFORD, A.E., DICKISON, W.C., MASSEY, J.R. \& BELL, C.R. 1974. Vascular Plants Systematic. Harper \& Row, New York.

RAPINI, A. 2012. Taxonomy "under construction":advances in the systematics of Apocynaceae, with emphasis on the Brazilian Asclepiadoideae. Rodriguésia 63(1):75-88, http://dx.doi.org/10.1590/ S2175-78602012000100007

RAPINI, A., KOCH, I., KINOSHITA, L.S., SIMÕES, A.O. \& SPINA, A.P. 2010. Apocynaceae. In Catálogo de Plantas e Fungos do Brasil (R.C. Forzza et al., eds.). Vol. 1. Andrea Jakobsson Estúdio/Instituto de Pesquisas Jardim Botânico do Rio de Janeiro, Rio de Janeiro, p.617-644.

RAPINI, A., KOCH, I., VALENTE, A.S.M., FERNANDEZ, E.P., MONTEIRO, N.P. \& MESSINA, T. 2013. Apocynaceae. In Livro 
Vermelho da Flora do Brasil (G. Martinelli \& M.A. Moraes, eds.). Andrea Jakobsson/Instituto de Pesquisas Jardim Botânico do Rio de Janeiro, Rio de Janeiro, p.157-174.

RIBEIRO, J.E.L.S., HOPKINS, M.J.G., VICENTINI, A., SOTHERS, C.A., COSTA, MA.S., BRITO, J.M., SOUZA, M.A.D., MARTINS, L.H.P., LOHMANN, L.G., ASSUNÇÃO, P.A.C.L., PEREIRA, E., SILVA, C.F., MESQUITA, M.R. \& PROCÓPIO, L.C. 1999. Flora da Reserva Ducke: guia de identificação das plantas vasculares de uma floresta de terra-firme na Amazônia Central. INPA/DFID, Manaus.

RIZZINI, C.T. 1977. Sistematização terminológica da folha. Rodriguésia 29(42):103-125.

SCHUMANN, K. 1895. Apocynaceae. In Die Natürlichen Pflanzenfamilien (A. Engler \& K. Prantl, eds.). W. Engelmann, Leipzig, teil 4, abt. 1-2, p.109-189.

SILVA, S. 2013. Árvores nativas do Brasil. Vol. 1. Editora Europa, São Paulo.

SIMÕES, A.O. \& KINOSHITA, L.S. 2002. The Apocynaceae s.str. of the Carrancas region, Minas Gerais, Brazil. Darwiniana 40(1-4):127-169.
SOUZA, C.M., VERÍSSIMO, A. \& AMARAL, P.H. 2002. Identificação de áreas com potencial para criação de florestas nacionais no Estado do Pará. Ministério do Meio Ambiente, Brasília.

STAFLEU, F.A. \& COWAN, R.S. 1981. Taxonomic literature: a selective guide to botanical publications and collections with dates, commentaries and types. Vol. 3. Bohn, Scheltema \& Holkema, Utrecht.

THIERS, B. [continuously updated]. Index Herbariorum: a global directory of public herbaria and associated staff. New York Botanical Garden's Virtual Herbarium. http://sweetgum.nybg.org/ih/ (last accessed on 01/05/2015).

WCSP - WORLD CHECKLIST OF SELECTED PLANT FAMILIES. 2015. World Checklist of Selected Plant Families. Facilitated by the Royal Botanic Gardens, Kew. http://apps.kew.org/wcsp/ home.do (last accessed on 01/05/2015).

WOODSON, R.E. 1951. Studies in the Apocynaceae VIII: an interim revision of the genus Aspidosperma Mart. \& Zucc. Ann. Missouri Bot. Gard. 38(1):119-204, http://dx.doi.org/10.2307/2394433

Received 28/06/2015

Revised 15/02/2016

Accepted 29/03/2016 